\title{
Diversidad de mariposas diurnas (Lepidoptera: Nymphalidae) en bosques y cultivos de cacao en un área de bosque amazónico basimontano en Bolivia
}

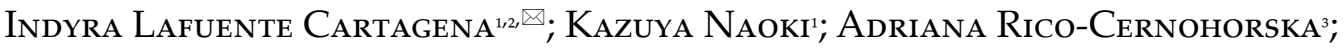 \\ Fernando Guerra-Serrudo ${ }^{3} \&$ Luis F. PACheCO ${ }^{3}$
}

\author{
${ }^{1}$ Instituto de Ecología, Carrera de Biología, Universidad Mayor de San Andrés (UMSA). La Paz-Bolivia. ${ }^{2}$ Asociación Boliviana \\ para la Investigación y Conservación de Ecosistemas Andino-Amazónicos (ACEAA-Conservación Amazónica). La Paz, Bolivia. \\ ${ }^{3}$ Colección Boliviana de Fauna, Instituto de Ecología, Carrera de Biología, Universidad Mayor de San Andrés (UMSA). La \\ Paz-Bolivia.
}

\begin{abstract}
RESUMEN. La conversión de bosques tropicales hacia la agricultura ha aumentado en los últimos años, aunque muchas veces ese cambio de uso del suelo es seguido por el abandono de los cultivos y su posterior conversión hacia bosques secundarios. En este estudio evaluamos el efecto de la perturbación sobre el ensamblaje de lepidópteros de la familia Nymphalidae en un área de bosque basimontano en Bolivia, para lo cual comparamos la diversidad y composición de mariposas entre bosques primarios, bosques secundarios y cultivos de cacao. Los datos se obtuvieron en época seca y en época de transición seca-húmeda por dos métodos de muestreo: red entomológica y trampeo con cebos. Registramos mayor abundancia, riqueza y heterogeneidad de especies de mariposas diurnas en la época seca. La riqueza de especies y la abundancia de mariposas en el sotobosque del bosque primario fue mayor que en cultivos durante la época de transición. El método de muestreo puede influir en la detección de cambios en las comunidades, ya que con trampas sólo detectamos variación estacional, mientras que con el muestreo manual registramos también mayor abundancia en ambos tipos de bosque que en los cultivos, y la heterogeneidad fue mayor en bosque primario que en cultivos. También se observó diferencia en la composición del ensamblaje de mariposas entre cultivos y ambos tipos de bosque. Aunque algunas mariposas de sotobosque son capaces de habitar sitios perturbados, es necesario resaltar la importancia del bosque primario para el mantenimiento de especies y grupos específicos que podrían perderse si la dinámica de perturbación se intensifica. Finalmente, es clave realizar estudios a largo plazo para entender la dinámica de las especies en la zona, tanto su estacionalidad como la relación con los recursos disponibles en cada tipo de hábitat.
\end{abstract}

[Palabras clave: bosque primario, bosque secundario, composición, Papilionoidea, red entomológica, trampa de cebo, GLMM, ordenamiento]

\begin{abstract}
Aвstract. Diversity of diurnal butterflies (Lepidoptera: Nymphalidae) in forests and cacao crops in an Amazonian-foothill forest in Bolivia. Tropical forests conversion to agriculture has increased in recent years, though often followed by land abandonment and subsequent conversion to secondary forest. In this study, we evaluated the effect of disturbance on Nymphalid butterfly assemblages in an area of mountain foothill forests in Bolivia by comparing the butterfly diversity and species composition among primary forests, secondary forests and cacao crops. Data were obtained in two seasons, dry and dry-to-wet transition, using two sampling methods (bait traps and hand net). Species richness and butterfly abundance were higher in the understory of primary forests than in crops during the transition season. The sampling method can influence the detection of community variation: with traps, we only detected seasonal variation, while we registered higher abundance in both types of forests than crops with manual sampling, with higher heterogeneity in the understory of primary forests than in crops. There was also a difference in butterfly species composition between crops and both types of forest. Although some understory butterflies are capable of inhabiting disturbed sites, we should highlight the importance of primary forest for the maintenance of specific species and groups that could be lost if the disturbance dynamics intensify. Finally, long-term studies are necessary to understand species dynamics in the area, both their seasonality and the interrelationship with available resources in each type of habitat.
\end{abstract}

[Keywords: Primary forest, secondary forest, composition, Papilionoidea, hand net, bait trap, GLMM, ordination] 


\section{INTRODUCCIÓN}

Uno de los mayores retos de la conservación es lograr su integración con el uso de la tierra (Kremen 1992; Gascon et al. 1999; Koh 2007). Esto es más evidente para los bosques tropicales, cuya extensión se ha reducido a causa de la deforestación generada por el cambio de uso de suelo (Brown and Lugo 1990; Wright and Muller-Landau 2006; Hansen et al. 2013). Por ejemplo, Bolivia es uno de los 10 países con mayor tasa de deforestación en la última década, con una tasa promedio de pérdida de bosque de 225000 ha/año (FAO 2020a,b). La deforestación suele ir seguida por el abandono de tierras de cultivo, lo cual genera mosaicos de zonas agrícolas, bosques secundarios y bosques maduros (Corlett 1995; Guariguata and Ostertag 2001; Wright 2005). Estos cambios alteran la disponibilidad de recursos y pueden afectar la composición y la dinámica de las comunidades en diferente grado según la intensidad y la persistencia de la modificación o perturbación (Corlett 1995; Perfecto et al. 1996; Mendenhall et al. 2014). El efecto de distintos grados de perturbación sobre la biodiversidad varía dependiendo del tipo de perturbación y del grupo taxonómico estudiado (Lawton et al. 1998; Harvey et al. 2006; Kessler et al. 2009). En los estudios realizados con mariposas, generalmente los sitios con perturbación baja tienen mayor abundancia y riqueza de especies (Veddeler et al. 2005; Barlow et al. 2007), pero ocasionalmente se ha reportado el incremento de estas variables en ambientes perturbados (Araujo 2000; Horner-Devine et al. 2003; González-Valdivia et al. 2016).

Las mariposas de la familia Nymphalidae son buenas indicadoras de perturbación en bosques tropicales, pues son muy sensibles a los cambios en la temperatura y la humedad. Su taxonomía y ecología están relativamente bien estudiadas (Kremen et al. 1993; DeVries et al. 1997; Warren et al. 2018) y pueden reflejar de forma adecuada la respuesta de otras familias de mariposas (Beccaloni and Gaston 1995). Aunque Nymphalidae suele tener dinámicas estacionales marcadas en respuesta a las variaciones en las condiciones ambientales de temperatura, precipitación y disponibilidad de recursos (DeVries and Walla 2001; Grøtan et al. 2012, 2014), se conoce poco sobre el efecto de la variación estacional bajo diferentes niveles de perturbación (Altermatt 2012; Lourenço et al. 2019). La mayoría de los estudios que evalúan el efecto de la perturbación sobre mariposas de la familia
Nymphalidae han utilizado el muestreo con trampas para mariposas frugívoras por la practicidad del método. En consecuencia, el efecto sobre las mariposas nectarívoras es poco conocido (Schulze et al. 2004; Harvey et al. 2006), ya que la respuesta a la perturbación puede ser diferente según el gremio alimentario de los organismos (Schulze et al. 2004; Harvey et al. 2006; Vargas et al. 2008). Si bien la abundancia, la riqueza de especies y la diversidad facilitan la comparación entre sitios bajo diferente uso o manejo, también se debe comparar la composición de especies (Mackey and Currie 2001; Hill and Hamer 2004), ya que las comunidades se podrían homogeneizar en ambientes perturbados a través del incremento de especies generalistas y la pérdida de especies o grupos funcionales raros o especialistas. Esto, a su vez, afectaría procesos ecológicos e interacciones importantes como la polinización, la depredación o el mantenimiento de la biodiversidad per se (Ghazoul 2002; Ekroos et al. 2010; Mendenhall et al. 2014; Supp and Ernest 2014).

El objetivo de este estudio fue comparar la riqueza y composición de los ensamblajes de mariposas Nymphalidae entre tres niveles de perturbación, dos épocas del ciclo hidrológico y dos métodos de muestreo en un bosque tropical basimontano en La Paz, Bolivia. Específicamente, nos preguntamos: 1) ¿Cuál es la variación en la estructura y composición del ensamblaje de mariposas asociada a la perturbación generada por bosques secundarios y cultivos de cacao respecto a los bosques primarios?, 2) ¿Cómo afecta la época del año a la respuesta de las comunidades de mariposas a la perturbación?, y 3) ¿Las conclusiones dependen del método de muestreo utilizado? Suponiendo que la pérdida de cobertura vegetal causada por la perturbación reduce la diversidad de las comunidades de mariposas, esperábamos encontrar menor riqueza y heterogeneidad de especies en sitios con perturbación alta (cultivos de cacao). Por otro lado, si se cumple que la variación estacional de las poblaciones de mariposas responde al cambio de las condiciones ambientales, entonces las diferencias entre niveles de perturbación deberían ser más marcadas durante la época de menor abundancia de recursos, ya que la baja heterogeneidad de recursos en los cultivos influiría negativamente en la preferencia de mariposas por este hábitat. Finalmente, si la perturbación afecta en diferente grado a distintos gremios alimentarios, entonces los resultados obtenidos con cebos para 
capturar principalmente un gremio (mariposas frugívoras) podrían diferir de los obtenidos por un método generalista (redes entomológicas).

\section{Materiales y Métodos}

\section{Área de estudio}

El estudio se realizó en la finca experimental agroecológica Sara Ana (15⒉ $37^{\prime} 30^{\prime}$ S $\left.67^{\circ} 28^{\prime} 38^{\prime \prime} \mathrm{O}\right)$, ubicada en el municipio Alto Beni, departamento La Paz, Bolivia (Figura 1). Sara Ana se encuentra entre 370 y 500 m s. n. m. La precipitación promedio anual es $1500 \mathrm{~mm}$ (SENAMHI 2020), con un pico de precipitación entre los meses diciembre y marzo, mientras que la época de menor precipitación corresponde a los meses de junio a agosto. La temperatura media anual es $26.6{ }^{\circ} \mathrm{C}$, con una disminución en los meses de mayo a agosto $\left(25^{\circ} \mathrm{C}\right)$ y máximas $\left(>27^{\circ} \mathrm{C}\right)$ entre septiembre y abril. La vegetación natural es bosque siempre verde estacional de llanura y pie de monte (Mueller et al. 2002; Campos et al. 2010). La composición florística es de transición entre Yungas y Amazonía, y comprende un mosaico de bosque relativamente bien conservado, pastizales y cultivos. La principal actividad económica en la zona es la agricultura, donde el cacao (Theobroma cacao) es el cultivo más frecuente.

\section{Muestreo de mariposas}

Se identificaron tres niveles de perturbación, tomando como criterio principal la composición de especies vegetales: 1) Bosque primario (perturbación baja), con alteraciones leves ocurridas hace más de 40 años (Seidel en prensa); presenta dosel de $20 \mathrm{~m}$ donde predominan arecáceas (Astrocaryum, Iriartea) y moráceas (Ficus, Clarisia) y se presentan varios subestratos, en los que se encuentran especies de los géneros Chamaedora (Araceae), Adiantum, Tectárea, Erythrochiton (Ruthacea), Carludovica (Cyclanthaceae), además de varias aráceas hemiepífitas (Monstera, Philodendron) y lianas como Dolichandra (Bignoniaceae) y Paullinia (Sapindaceae); 2) Bosque secundario (perturbación media); resultado de perturbación natural y antrópica del bosque con >25 años de abandono, con dominancia de especies de Cecropia (Urticaceae) y Triplaris (Polygonaceae); y 3) Cultivos de cacao (perturbación alta) acompañados de plátano (Musa spp., Musaceae) y con otras especies ocasionales como motacú (Attalea princeps, Arecaceae), ceibo (Erythrina poepiggiana,
Fabaceae), achiote (Bixa orellana, Bixaceae) y guayaba (Psidium guajava, Myrtaceae). A pesar de cierta variación entre ellos, en los análisis se utilizaron los datos de los cultivos sin distinción, ya que no resultaron separados según su composición de mariposas en los análisis de ordenamiento.

Se escogieron 10 sitios de muestreo por cada nivel de perturbación $(n=30)$ (Figura 1$)$, separados entre sí por al menos 120 m (mayor a la distancia mínima sugerida para lograr independencia en los datos en estudios de ensamblajes de mariposas [Hamer et al. 2003; Ribeiro et al. 2012]). El muestreo fue realizado en las épocas seca (agosto) y transición secahúmeda (noviembre-diciembre) de 2015 empleando capturas con trampas de cebo y con red entomológica. En cada sitio de muestreo se instaló una trampa de cebo para mariposas frugívoras tipo Van Someren-Rydon (cilindros de $\sim 1 \mathrm{~m}$ de altura que permiten el vuelo de las mariposas en su interior) (DeVries et al. 1997), colgada a $2 \mathrm{~m}$ de altura y cebada con una mezcla fermentada de frutas, atún, vísceras de pollo y cerveza. El cebo se puso un día antes de iniciar el muestreo y se remplazó cada dos días. Las trampas, que se revisaron cada 24 horas, estuvieron activas durante cinco días consecutivos de cada época en cada sitio de muestreo (total: 300 días-trampa). La captura manual se realizó con una red entomológica a lo largo de dos transectos de $50 \mathrm{~m}$ (separados entre sí por $15 \mathrm{~m}$ ) en cada sitio de muestreo. Cada transecto fue recorrido durante 10 minutos dos veces en cada época, una vez por la mañana y una por la tarde, siempre por la misma persona (IL) (total: 2400 minutos de muestreo). Los individuos recolectados durante ambos recorridos por cada transecto fueron agregados (es decir, se obtuvo un dato por época y sitio). Todos los individuos fueron colectados mediante presión torácica y guardados en sobres entomológicos para su posterior identificación. La identificación de especies fue realizada por comparación con especímenes de la Colección Boliviana de Fauna (CBF) y las guías de Gareca et al. (2006) y Warren et al. (2018). La nomenclatura sigue la propuesta de Lamas et al. (2004, citado en Warren et al. 2018). Los especímenes colectados fueron depositados en la CBF.

\section{Estimación de la cobertura vegetal}

En cada sitio de muestreo se registraron los porcentajes de cobertura herbácea y arbustiva mediante el método de línea-intercepción (Matteucci and Colma 1982) en un transecto de 


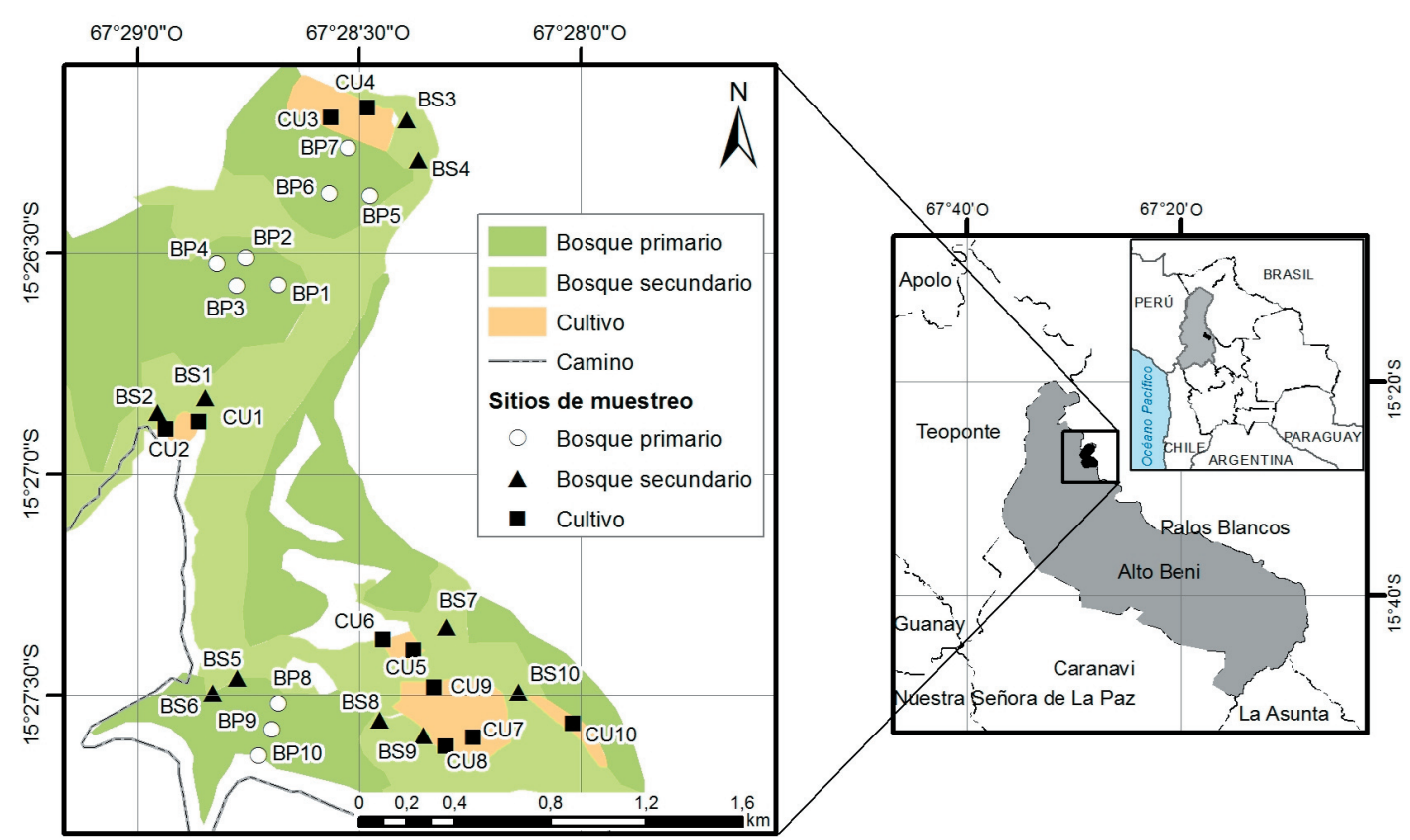

Figura 1. Ubicación geográfica del área de estudio y de los 30 sitios de muestreo dentro del Departamento La Paz, Bolivia.

Figure 1. Geographical location of study area and the 30 sampling sites within La Paz, Bolivia.

$20 \mathrm{~m}$, considerando como cobertura arbustiva a las plantas $>1.5 \mathrm{~m}$ de altura. El porcentaje de cobertura de dosel se estimó como el promedio de 10 observaciones puntuales realizadas cada $2 \mathrm{~m}$ sobre el mismo transecto; en cada observación se estimó el porcentaje de cobertura mediante observación directa hacia el cielo usando un cilindro de $5 \mathrm{~cm}$ de diámetro y $10 \mathrm{~cm}$ de largo.

\section{Análisis de datos}

Los datos obtenidos por ambos métodos de muestreo de mariposas se complementan y permiten tener una lista más completa de especies de Nymphalidae (Wood and Gillman 1998; Castro and Espinosa 2015; Jakubikova and Kadlec 2015; Graça et al. 2017), pero no se pueden combinar para estimar la abundancia de especies. Por tanto, los índices que no requieren información de abundancia, como riqueza y lista de especies, se elaboraron para cada sitio y época utilizando los datos de ambos métodos de muestreo integrados, mientras que la abundancia y heterogeneidad (inverso del índice de diversidad de Simpson [Simpson 1949]) se calcularon de manera separada para cada método de muestreo. La suficiencia del esfuerzo de muestreo fue evaluada mediante curvas de rarefacción (no incluidas en el artículo) y la comparación del número de especies registrado con el número estimado por el índice de Chao 1 bajo cada nivel de perturbación (Chiu et al. 2014).

Para evaluar los efectos del nivel de perturbación, la cobertura vegetal y la época del año sobre los ensamblajes de mariposas, se utilizaron modelos lineales generalizados de efectos mixtos (GLMM) con la riqueza de especies, la abundancia y la heterogeneidad como variables dependientes; el nivel de perturbación, la época de muestreo y los porcentajes de cobertura vegetal como las variables independientes potenciales de efecto fijo, y el sitio de muestreo como efecto aleatorio (Faraway 2016). Se evaluó la multicolinealidad entre las variables independientes potenciales por el diagrama de dispersión matricial y los factores de inflación de la varianza (VIF). Al encontrar multicolinealidad se eliminó el porcentaje de la cobertura dosel, que estuvo correlacionado con el nivel de perturbación, quedando las siguientes variables en los modelos globales: el nivel de perturbación (3 niveles), la época del año (2 niveles), los porcentajes de la cobertura herbácea y de la cobertura arbustiva y los sitios de muestreo. Se utilizaron los GLMM con la distribución gaussiana y la función de enlace identity para modelar el índice de heterogeneidad, y con la distribución Poisson y el enlace log para la riqueza de especies y la abundancia. Se evaluó el ajuste de la distribución de residuos a los 
supuestos de los modelos por medio del gráfico Q-Q, el gráfico de residuos y el parámetro de dispersión. Cuando se detectó un desajuste (e.g., por ejemplo, sobredispersión), se aplicaron otras distribuciones de errores y funciones de enlace (Richards 2008).

Para seleccionar las variables independientes incluidas en el modelo se crearon los modelos parciales mediante la simplificación del modelo global que contenía todas las variables independientes no colineales. Se realizó la comparación de los modelos por el criterio de información de Akaike corregido para muestras pequeñas (AICc, Burnham and Anderson 2002), y todos los modelos con delta AICc $<2$ (delta AICc=AICc del modelo focal-AICc anidado más pequeño) fueron presentados como los mejores modelos. Adicionalmente, se calculó la importancia relativa (IR) de cada variable independiente como la suma del peso de Akaike de todos los modelos que incluyeron la variable de interés (Giam and Olden 2016, pero ver Galipaud et al. 2017). Los parámetros de cada variable independiente fueron estimados y su significancia estadística fue determinada en función de la tabla de resumen (summary) del mejor modelo con el AICc más pequeño. Se compararon las variables dependientes entre los niveles de perturbación mediante pruebas post hoc de Tukey cuando se detectó efecto de la perturbación; este análisis se realizó separadamente para cada época cuando además se detectó efecto de interacción entre la perturbación y la época.

La variación espacial y temporal en la composición de mariposas fue analizada por dos métodos: 1) el ordenamiento indirecto de la lista de especies de cada sitio para reducir la dimensión de los datos altamente multivariados y la prueba estadística de las primeras dimensiones de ordenamiento. Inicialmente, la ordenación se realizó por el análisis de correspondencia (CA); sin embargo, el gráfico de los dos primeros ejes mostró el efecto de arco y no fue posible interpretar la segunda dimensión. Así, se utilizó el análisis de correspondencia rectificado (DCA; Hill and Gauch 1980), elcual permiteextraer los patrones dominantes de variación en la composición de los ensambles biológicos (Jongman et al. 1995). Luego se utilizaron los modelos lineales generalizados de efecto mixto (GLMM) para evaluar los efectos del nivel de perturbación, la cobertura vegetal y la época del año sobre las primeras dos dimensiones de DCA. 2) El ordenamiento directo de la presencia-ausencia de cada especie bajo diferentes condiciones ambientales (e.g., Hoy et al. 2008), mediante el análisis de correspondencia canónica (CCA), en el cual se analizaron los efectos del nivel de perturbación, la cobertura herbácea, la cobertura arbustiva, la época de año y la estructura espacial de los sitios de muestreo. Esta última fue cuantificada por medio del mapa del eigenvector de Moran (MEM) (Dray et al. 2006). En el CCA se realizó la selección hacia delante (forward selection) para seleccionar las variables independientes importantes. La contribución relativa de los tres grupos de las variables independientes (nivel de perturbación, época y estructura espacial) se analizó por la partición de variación (Borcard et al. 2018).

Todos los análisis estadísticos se realizaron con el programa $\mathrm{R}$ (versión 3.6.2 [R Core Team 2019]), con el paquete vegan para el cálculo del índice de diversidad y Chao, DCA y CCA (Oksanen et al. 2019), el paquete car para calcular VIFs (Fox and Weisberg 2019), el paquete lme4 para ajustar GLMM (Bates et al. 2015), el paquete MASS para GLMM con la distribución binomial negativa (Venables and Ripley 2002), el paquete MuMIn para calcular AICc, comparar los modelos y estimar el $R^{2}$ marginal (Barton 2019), el paquete multcomp para la comparación post hoc (Hothorn 2008) y el paquete adespatial para realizar el MEM (Dray et al. 2019).

\section{Resultados}

Se registraron 761 mariposas diurnas de la familia Nymphalidae correspondientes a 107 especies y ocho subfamilias. El número de individuos capturados fue bastante similar entre niveles de perturbación, pero fue casi el doble en época seca que en época de transición seca-húmeda (Tabla 1). La abundancia promedio $( \pm \mathrm{DE})$ de individuos por especie registrada fue $7.1 \pm 8.6$ (rango: $1-47$ ). Veinticinco especies (23\%) estuvieron representadas sólo por un individuo y 17 especies (16\%) por dos individuos. La abundancia registrada en cada muestreo varió entre 0 y 23 individuos (6.3ะ4.8; con solo 9 de las 120 muestras sin capturas) y la riqueza de especies entre 0 y $15(4.4 \pm 3.0)$.

\section{Riqueza de especies}

La riqueza de especies observada fue similar entre bosque primario y cultivo y representó entre el 76 y $81 \%$ de la riqueza total esperada 
Tabla 1. Abundancia (número de individuos) y riqueza de especies registradas en los 10 sitios bajo cada uno de los tres niveles de perturbación estudiados, en ambas épocas de muestreo y por ambos métodos. Se presenta también la riqueza de especies esperada (según el índice de Chao 1) para cada nivel de perturbación integrando ambos métodos de captura y épocas.

Table 1. Abundance (number of individuals) and species richness registered in the 10 sites of each studied disturbance level (primary forests, secondary forests and crops) in both sampling seasons (dry and dry-humid transition) and for both sampling methods (baited traps and entomological nets). The predicted species richness (according to Chao 1 index) for each disturbance level, aggregating both seasons and trapping methods, is also presented.

\begin{tabular}{|c|c|c|c|c|c|c|c|c|c|}
\hline & \multirow{2}{*}{$\begin{array}{c}\text { Nivel de } \\
\text { perturbación }\end{array}$} & \multicolumn{3}{|c|}{ Seca } & \multicolumn{3}{|c|}{ Transición } & \multirow{2}{*}{ TOTAL } & \multirow{2}{*}{$\begin{array}{c}\text { Índice de } \\
\text { Chao }\end{array}$} \\
\hline & & Trampa & Red & Total & Trampa & Red & Total & & \\
\hline \multirow{4}{*}{$\begin{array}{l}\text { Riqueza de } \\
\text { especies }\end{array}$} & Bosque primario & 28 & 20 & 42 & 24 & 26 & 48 & 63 & $77.7 \pm 8.2$ \\
\hline & Bosque secundario & 30 & 37 & 56 & 18 & 30 & 40 & 71 & $90.7 \pm 10.0$ \\
\hline & Cultivo & 32 & 31 & 51 & 21 & 12 & 29 & 62 & $82.3 \pm 10.7$ \\
\hline & TOTAL & 55 & 58 & 89 & 39 & 54 & 75 & 107 & \\
\hline
\end{tabular}

en cada nivel de perturbación (según el índice de Chao 1) (Tabla 1). El mejor modelo explicativo de la riqueza de especies incluyó a la época (importancia relativa: IR=1.00), el nivel de perturbación $(I R=0.59)$ y a su interacción ( $\mathrm{IR}=0.53$ ) (distribución Poisson con el enlace log: $R_{\text {marginal }}^{2}=0.35$ ) (Tabla 2). La riqueza de especies fue menor en época transición que en seca (Figura 2a) $(Z=4.7, P<0.0001)$. La riqueza de especies no varió entre los niveles de perturbación durante la época seca (Tukey: $P>0.3$ para todas las comparaciones), pero fue mayor en los bosques primarios que en los cultivos durante la época de transición (Tukey: $Z=2.7, P=0.019$ ).

\section{Abundancia}

Se observó mayor abundancia de mariposas capturadas con trampas de cebo durante la época seca que en transición (distribución binomial negativa con el enlace $\log$ : $\mathrm{IR}=0.92$, $R_{\text {marginal }}^{2}=0.08$ ) (Tabla 2). La abundancia de mariposas capturadas con red entomológica

Tabla 2. Resumen de los resultados de los GLMM con la riqueza de especies, la abundancia (estimada por dos métodos de trampeo: red entomológica y trampa de cebo), la heterogeneidad (inverso del índice de Simpson) y la composición como variables dependientes, y la época (Epc, 2 niveles), el nivel de perturbación (Per, 3 niveles), la interacción entre ambos, la cobertura arbustiva (Arb) y la cobertura herbácea (Herb) como variables independientes. Todos los

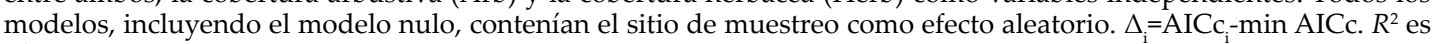
$R^{2}$ marginal.

Table 2. Summary of the GLMM results with species richness, abundance (estimated by two trapping methods: entomological net and bait trap), heterogeneity (inverse of the Simpson index) and composition as dependent variables, and season (Epc, 2 levels), disturbance level (Per, 3 levels), interaction between both, shrub cover (Arb) and herbaceous cover (Herb) as independent variables. All models, including the null model, contained the sampling site as the independent variable of random effect. $\Delta_{\mathrm{i}}=$ AIC $\mathrm{C}_{\mathrm{i}}$-min AICc. $R^{2}$ is marginal $R^{2}$.

\begin{tabular}{|c|c|c|c|c|c|}
\hline Variable dependiente & $\begin{array}{l}\text { Mejores modelos }(\triangle \mathrm{AICc}<2) \\
\text { y modelo nulo }\end{array}$ & AICc & g.l. & $\Delta_{\mathrm{i}}$ & $R^{2}$ \\
\hline Riqueza & $\begin{array}{l}\text { Epc }+ \text { Per }+ \text { Epc*Per } \\
\text { Epc } \\
\text { Nulo }\end{array}$ & $\begin{array}{l}337.3 \\
338.9 \\
359.6\end{array}$ & $\begin{array}{l}7 \\
3 \\
2\end{array}$ & $\begin{array}{c}0 \\
0.7 \\
22.3\end{array}$ & $\begin{array}{l}0.35 \\
0.24\end{array}$ \\
\hline Abundancia (trampa) & $\begin{array}{l}\text { Epc } \\
\text { Epc + Herb } \\
\text { Epc + Arb } \\
\text { Nulo }\end{array}$ & $\begin{array}{l}341.7 \\
342.4 \\
343.7 \\
347.4\end{array}$ & $\begin{array}{l}4 \\
5 \\
5 \\
3\end{array}$ & $\begin{array}{l}0 \\
0.7 \\
1.9 \\
5.7\end{array}$ & $\begin{array}{l}0.08 \\
0.09 \\
0.08\end{array}$ \\
\hline Abundancia (red) & $\begin{array}{l}\text { Epc }+ \text { Per }+ \text { Epc*Per } \\
\text { Nulo }\end{array}$ & $\begin{array}{l}332.5 \\
367.0\end{array}$ & $\begin{array}{l}7 \\
2\end{array}$ & $\begin{array}{c}0 \\
34.4\end{array}$ & 0.39 \\
\hline Heterogeneidad (trampa) & $\begin{array}{l}\text { Epc } \\
\text { Epc }+ \text { Per }+ \text { Epc }{ }^{*} \text { Per } \\
\text { Epc }+ \text { Per } \\
\text { Nulo }\end{array}$ & $\begin{array}{l}298.5 \\
298.8 \\
299.8 \\
303.1\end{array}$ & $\begin{array}{l}4 \\
8 \\
6 \\
3\end{array}$ & $\begin{array}{c}0 \\
0.3 \\
1.3 \\
4.7\end{array}$ & $\begin{array}{l}0.06 \\
0.09 \\
0.07\end{array}$ \\
\hline Heterogeneidad (red) & $\begin{array}{l}\text { Epc }+ \text { Per }+ \text { Epc }^{*} \text { Per } \\
\text { Nulo }\end{array}$ & $\begin{array}{l}253.1 \\
261.7\end{array}$ & $\begin{array}{l}8 \\
3\end{array}$ & $\begin{array}{c}0 \\
8.6\end{array}$ & 0.21 \\
\hline Composición (DCA1) & $\begin{array}{l}\text { Per } \\
\text { Nulo }\end{array}$ & $\begin{array}{l}157.3 \\
173.4\end{array}$ & $\begin{array}{l}5 \\
3\end{array}$ & $\begin{array}{c}0 \\
16.2\end{array}$ & 0.43 \\
\hline Composición (DCA2) & $\begin{array}{l}\text { Epc }+ \text { Per }+ \text { Epc } c^{*} \text { Per } \\
\text { Nulo }\end{array}$ & $\begin{array}{l}155.8 \\
170.9\end{array}$ & $\begin{array}{l}8 \\
3\end{array}$ & $\begin{array}{c}0 \\
15.0\end{array}$ & 0.39 \\
\hline
\end{tabular}


a)

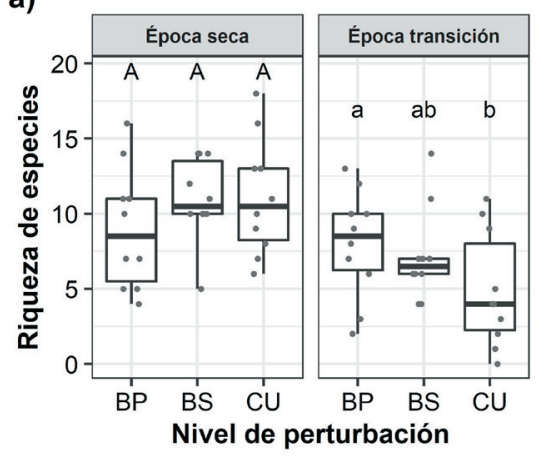

b)

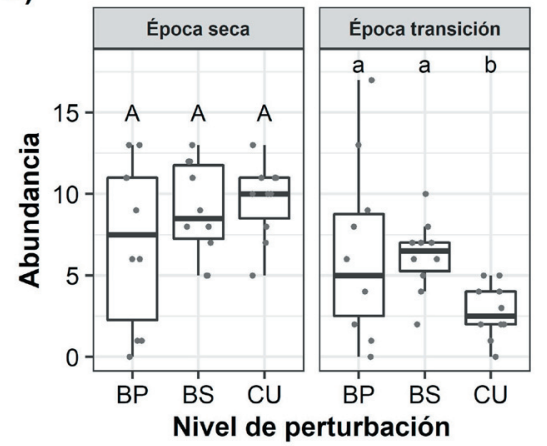

c)

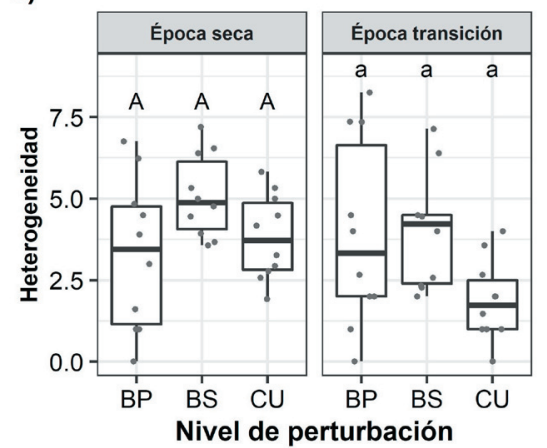

Figura 2. Diagramas de caja y bigote mostrando los efectos de la época y el nivel de perturbación sobre los ensamblajes de mariposas: (a) riqueza de especies, (b) abundancia de mariposas capturadas con red entomológica y (c) heterogeneidad de mariposas capturadas con red entomológica. Las cajas representan el rango intercuartílico, las líneas centrales representan la mediana y los bigotes representan 1.5 veces el rango intercuartílico. Los puntos correspondientes a cada sitio de muestreo se presentan fluctuados (jitter) en sentido horizontal. $\mathrm{BP}=$ bosque primario, $\mathrm{BS}=$ bosque secundario, $\mathrm{CU}=$ cultivo de cacao. Letras diferentes sobre las cajas indican diferencia significativa por la comparación de Tukey.

Figure 2. Boxplots showing the effects of season and disturbance level on butterfly assemblages: (a) species richness, (b) abundance of butterflies captured by hand net, and (c) heterogeneity of butterflies captured by hand net. The boxes represent the interquartile range, the central lines represent the median, and the whiskers represent 1.5 times the interquartile range. Points representing values for each sampling site were plotted as jittered along the horizontal axis. $\mathrm{BP}=$ primary forest, $\mathrm{BS}=$ secondary forest, $\mathrm{CU}=$ cacao crop. Different letters above the boxes indicate significant differences in Tukey test. varió según la época $(\mathrm{IR}=1.00)$, el nivel de perturbación $(\mathrm{IR}=0.99)$ y su interacción $(\mathrm{IR}=0.99)$ (distribución Poisson con el enlace log: $R_{\text {marginal }}^{2}=0.39$ ) (Figura 2b, Tabla 2). Se registraron más mariposas en época seca que en transición (Tabla 1). La abundancia no varió entre los niveles de perturbación durante la época seca $(P>0.2$ para todas las comparaciones), pero fue mayor en los bosques que en cultivos durante la época de transición (Tukey: BP vs. $\mathrm{CU}, \mathrm{Z}=2.7, \mathrm{P}=0.018$; $\mathrm{BS}$ vs. $\mathrm{CU}, \mathrm{Z}=2.6, P=0.025)$.

\section{Heterogeneidad}

La heterogeneidad de mariposas capturadas con trampas de cebo solo varió entre las dos épocas ( $\mathrm{IR}=0.94)$, con mayor heterogeneidad durante la época seca (distribución gaussiana con el enlace identity: $R_{\text {marginal }}^{2}=0.06$ ) (Tabla 2). La heterogeneidad de mariposas registradas con red entomológica varió según la época $(\mathrm{IR}=0.97)$, el nivel de perturbación $(\mathrm{IR}=0.94) \mathrm{y}$ su interacción $(\mathrm{IR}=0.84)$ (distribución gaussiana con el enlace identity: $R_{\text {marginal }}^{2}=0.21$ ) (Figura 2c, Tabla 2); siendo mayor durante la época seca que en la época de transición $\left(t_{27}=2.8, P=0.01\right)$, aunque la heterogeneidad no varió entre los niveles de perturbación durante cada época por la comparación post hoc (Tukey: $P>0.05$ para todas las comparaciones, aunque estuvieron cercanas a la significancia estadística).

\section{Composición del ensamblaje}

La composición del ensamblaje de mariposas varió entre los niveles de perturbación: los sitios con bosques (BP+BS) y con cultivos (CU) se pueden separar en el plano dado por los dos primeros ejes del DCA (aunque con bastante solapamiento) (Figura 3). DCA1 varió según el nivel de perturbación (distribución gaussiana con el enlace identity: $\left.\mathrm{IR}=1, R_{\text {marginal }}^{2}=0.43\right) \mathrm{y}$ DCA2 según la época ( $I R=1)$, el nivel ( $I R=0.99)$ y su interacción (distribución gaussiana con el enlace identity: $\mathrm{IR}=0.98, R_{\text {marginal }}^{2}=0.39$ ).

Las especies más abundantes en el sitio de estudio fueron Hermeuptychia sp. y Ceratinia neso, con 47 y 40 individuos, respectivamente. Durante la época seca, C. neso fue la más abundante, seguida por Hermeuptychia sp., con 34 y 26 individuos, respectivamente. Por otro lado, en época de transición, Hermeuptychia sp. fue la más abundante, seguida por Mechanitis polymnia, con 21 y 16 individuos, respectivamente. Ceratinia neso tuvo una reducción marcada de su abundancia en 


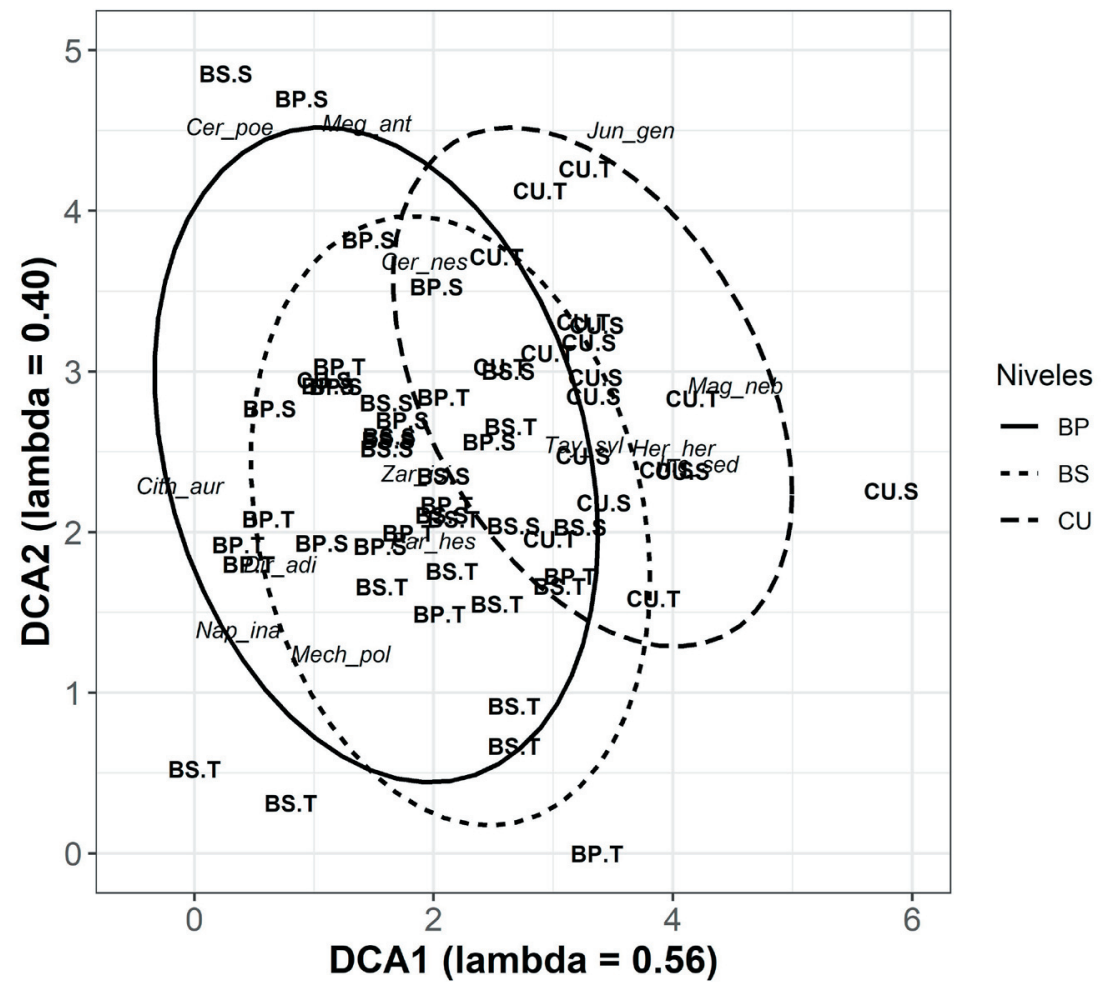

Figura 3. Ordenamiento de los sitios de muestreo según la lista de especies de mariposas por medio del análisis de correspondencia rectificado (DCA). $\mathrm{BP}=$ bosque primario, $\mathrm{BS}=$ bosque secundario, $\mathrm{CU}=$ cultivo, $\mathrm{S}=$ =época seca, $\mathrm{T}=$ =época transición. Las elipses indican las áreas de confianza de 95\% para cada hábitat. Ver Tabla 3 para las abreviaciones de los nombres de las especies de mariposas. Para facilitar la interpretación del gráfico sólo se muestran las especies más abundantes.

Figure 3. Detrended component analysis of sampling sites according to butterfly species list. BP=primary forest, $\mathrm{BS}=$ secondary forest, $\mathrm{CU}=$ cacao crops, $\mathrm{S}=\mathrm{dry}$ season, $\mathrm{T}=$ transition season. Ellipses indicate $95 \%$ confidence areas for each habitat. See Table 3 for butterfly species abbreviations. Only main species are presented to help interpretation and reduce clutter.

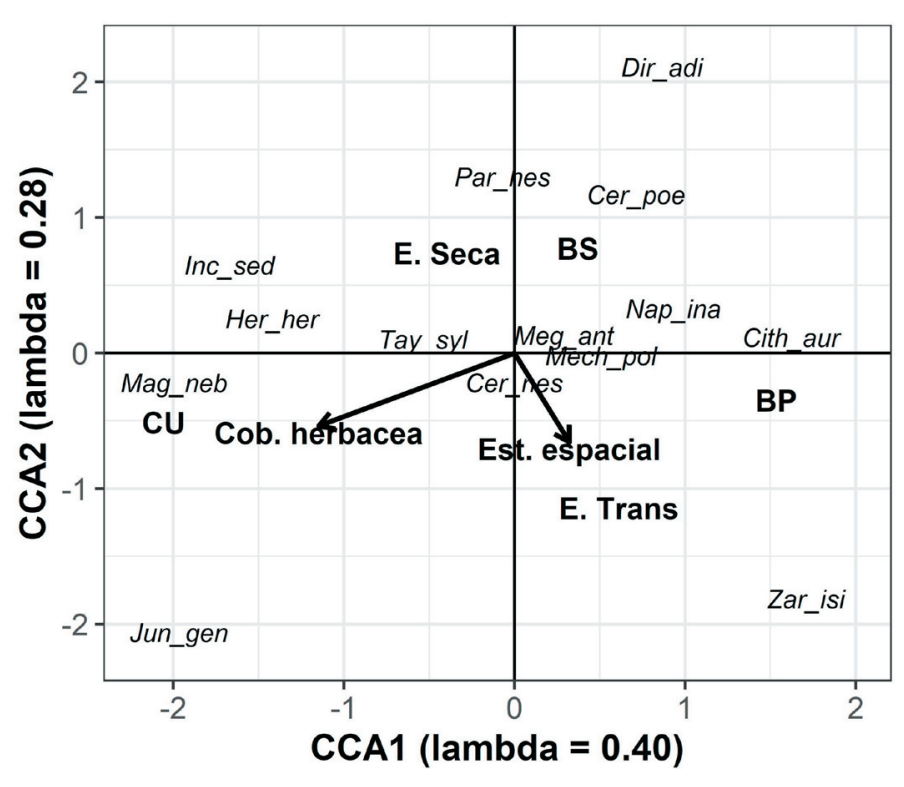

Figura 4. Ordenamiento de las especies de mariposas por medio del análisis de correspondencia canónica (CCA). $\mathrm{BP}=$ bosque primario, $\mathrm{BS}=$ bosque secundario, $C U=$ cultivo, E. Seca=época seca, E. Trans=época transición, Cob. Herbácea=porcentaje de la cobertura herbácea, Est. espacial=estructura espacial representada por el primer eje del mapa de eigenvector de Moran (MEM1). Ver Tabla 3 para las abreviaciones de especies de mariposas. Para facilitar la interpretación sólo se muestran las especies más abundantes.

Figure 4. Canonical correspondence analysis (CCA) of butterfly species. $\mathrm{BP}=$ primary forest, $\mathrm{BS}=$ secondary forest, $\mathrm{CU}=$ cacao crops, E. Seca=dry season, $\mathrm{E}$. Trans=dry-humid transition season, Cob. Herbácea=herbaceous cover percentage, Est. espacial=spatial structure represented by the first axis of Moran's eigenvector map (MEM1). See Table 3 for butterfly species abbreviations. Only main species are presented to help interpretation and reduce clutter. 
época de transición, con sólo 6 individuos registrados.

Según los análisis y la lista de especies (Tabla 3), dos especies (Cithaerias aurorina y Zaretis isidora) fueron comunes y exclusivamente registradas en bosque primario. Cuatro especies (Napeogenes inachia, Ceratinia poecilia, Memphis phantes y Pseudodebis valentina) fueron comunes en bosque primario, con algunos registros en bosque secundario y muy raras en los cultivos. Dos especies (Pareuptychia hesionides y M. polymnia) fueron comunes en bosque secundario, menos abundantes en bosque primario y muy raras en cultivos. Junonia genoveva fue común y exclusiva en los cultivos. Cuatro especies (Hermeuptychia hermes, Hermeuptychia. sp., Incertae sedis nerita y Magneuptychia nebulosa) fueron comunes en los cultivos, menos abundantes en bosque secundario y no fueron registradas en bosque primario. Siete especies (P. hesionides, C. poecilia,

Tabla 3. Presencia de especies capturadas por método manual de red entomológica (R) y trampa (T) en los 10 sitios bajo cada nivel de perturbación: bosque primario $(\mathrm{P})$, bosque secundario $(\mathrm{S})$ y cultivo $(\mathrm{C})$. Los nombres abreviados son referencia para las Figuras 3 y 4 .

Table 3. Presence of butterfly species captured using entomological net $(\mathrm{R})$ and trap sampling $(\mathrm{T})$ at the 10 sites under each disturbance level, primary forest $(\mathrm{P})$, secondary forest $(\mathrm{S})$, and crop $(\mathrm{C})$. Abbreviated names are shown as reference for Figures 3 and 4.

\begin{tabular}{|c|c|c|c|c|c|c|c|c|}
\hline \multirow{2}{*}{ Especie } & \multirow[b]{2}{*}{ Abreviatura } & \multicolumn{2}{|c|}{$\mathrm{BP}$} & \multicolumn{2}{|c|}{ BS } & \multicolumn{2}{|l|}{$\mathrm{C}$} & \multirow[b]{2}{*}{ Hábitat $^{1}$} \\
\hline & & $\mathrm{R}$ & $\mathrm{T}$ & $\mathrm{R}$ & $\mathrm{T}$ & $\mathrm{R}$ & $\mathrm{T}$ & \\
\hline \multicolumn{9}{|l|}{ Subfamilia Biblidinae } \\
\hline Biblis hyperia (Cramer 1779) & Byb_hyp & & $\mathrm{x}$ & & $\mathrm{x}$ & & $\mathrm{x}$ & AA, $P^{*}$ \\
\hline Callicore eunomina (Neild 1996) & Cal_eun & & $\mathrm{x}$ & $\mathrm{x}$ & & & & $\mathrm{CB}, \mathrm{BB}$ \\
\hline Catonephele acontius (Linnaeus 1771) & Cat_aco & & $\mathrm{x}$ & $\mathrm{x}$ & $\mathrm{x}$ & & & BS, D \\
\hline Catonephele salacia (Hewitson 1852) & Cat_sal & & $\mathrm{x}$ & $\mathrm{x}$ & $\mathrm{x}$ & $\mathrm{x}$ & & \\
\hline Diaethria clymena (Guérin-Méneville 1844) & Dia_cly & & & & & $\hat{x}$ & $\mathrm{x}$ & \\
\hline Diaethria kolyma (Hewitson 1852) & Dia_col & & & & & & $\mathrm{x}$ & $\mathrm{CB}, \mathrm{SB}$ \\
\hline Dynamine aerata (Butler 1877) & Dyn_aer & & & & & $\mathrm{x}$ & & $\mathrm{CB}, \mathrm{BB}$ \\
\hline Dynamine arene (Hübner 1823) & Dyn are & & & & $\mathrm{x}$ & $\mathrm{x}$ & & $\mathrm{P}$ \\
\hline Dynamine postverta (Cramer 1779) & Dyn_pos & & & & & $\mathrm{x}$ & & $\mathrm{P}$ \\
\hline Eunica alpais (Godart 1824) & Eun_alp & & & & & $\mathrm{x}$ & & AA \\
\hline Eunica amelia (Cramer 1777) & Eun_ame & & & & & $\mathrm{x}$ & & \\
\hline Eunica sophonisba (Cramer 1780) & Eun_sop & & & & & $\mathrm{x}$ & & $\mathrm{D}, \mathrm{BS}$ \\
\hline Eunica sydonia (Godart 1824) & Eun syd & & & $\mathrm{x}$ & & & & $\mathrm{BM}, \mathrm{BB}, \mathrm{AA}$ \\
\hline Hamadryas arinome (Lucas 1853) & Ham ari & & & & $\mathrm{x}$ & & & $\mathrm{BM}, \mathrm{CB}$ \\
\hline Hamadryas chloe (Stoll 1787) & Ham_chl & $\mathrm{x}$ & & & & & & BM, P \\
\hline Hamadryas epinome (Felder and Felder 1867) & Ham_epi & & $\mathrm{x}$ & & & & & $\mathrm{AA}, \mathrm{BM}$ \\
\hline Hamadryas februa (Hübner 1823) & Ham_feb & & & & & & $\mathrm{x}$ & AA, P \\
\hline Hamadryas feronia (Linnaeus 1758) & Ham fer & & $\mathrm{x}$ & & & $\mathrm{x}$ & $\mathrm{x}$ & AA, $\mathrm{P}^{*}$ \\
\hline Nessaea hewitsonii (Felder and Felder 1859) & Nes hew & & & & $\mathrm{x}$ & & & \\
\hline Nessaea obrinus (Linnaeus 1758) & Nes_obr & & $\mathrm{x}$ & & & & & $\mathrm{BM}^{*}$ \\
\hline Nica flavilla (Godart 1824) & Nic_fla & & & & $\mathrm{x}$ & & & $\mathrm{AA}, \mathrm{MA}^{*}$ \\
\hline Pyrrhogyra amphiro (Bates 1865) & Pyr_amp & & & & $\mathrm{x}$ & $\mathrm{x}$ & $\mathrm{x}$ & \\
\hline Pyrrhogyra crameri (Aurivillius 1882) & Pyr_cra & & $\mathrm{x}$ & $\mathrm{x}$ & $\mathrm{x}$ & & & $\mathrm{CB}$ \\
\hline Pyrrhogyra otolais (Fruhstorfer 1980) & Pyr oto & & $\mathrm{x}$ & $\mathrm{x}$ & $\mathrm{x}$ & $\mathrm{x}$ & & $\mathrm{CB}, \mathrm{AA}$ \\
\hline Temenis laothoe (Cramer 1777) & Tem_lao & & & $\mathrm{x}$ & $\mathrm{x}$ & & $\mathrm{x}$ & $\mathrm{CB}, \mathrm{P}^{*}$ \\
\hline \multirow{2}{*}{\multicolumn{9}{|c|}{$\begin{array}{l}\text { Vila azeca (Doubleday 1848) } \\
\text { Subfamilia Charaxinae }\end{array}$}} \\
\hline & & & & & & & & \\
\hline Consul fabius (Butler 1874) & Con_fab & & $\mathrm{x}$ & $\mathrm{x}$ & $\mathrm{x}$ & $\mathrm{x}$ & & $\mathrm{BM}^{*}$ \\
\hline Fountainea eurypyle (Felder and Felder 1862) & Fou eur & & $\mathrm{x}$ & & $\mathrm{x}$ & & $\mathrm{x}$ & \\
\hline Fountainea ryphea (Cramer 1775) & Fou_ryp & & $\mathrm{x}$ & & $\mathrm{x}$ & & $\mathrm{x}$ & BM, BS, P \\
\hline Fountainea sosippus (Hopffer 1874) & Fou_sos & & $\mathrm{x}$ & & & & $\mathrm{x}$ & \\
\hline Hypna clytemnestra (Cramer 1777) & Hyp_cly & $\mathrm{x}$ & $\mathrm{x}$ & & $\mathrm{x}$ & & & BS, D* \\
\hline Memphis acidalia (Hübner 1819) & Mem_aci & & $\mathrm{x}$ & & $\mathrm{x}$ & & $\mathrm{x}$ & $\mathrm{SB}, \mathrm{D}$ \\
\hline Memphis glauce (Möschier 1877) & Mem gla & & $\mathrm{x}$ & $\mathrm{x}$ & $\mathrm{x}$ & & $\mathrm{x}$ & $\mathrm{CB}$ \\
\hline Memphis phantes (Hopffer 1874) & Mem_pha & $\mathrm{x}$ & $\mathrm{x}$ & & $\mathrm{x}$ & & $\mathrm{x}$ & \\
\hline Memphis xenocles (Westwood 1850) & Mem_xen & & $\mathrm{x}$ & $\mathrm{x}$ & $\mathrm{x}$ & $\mathrm{x}$ & $\mathrm{x}$ & SB, D \\
\hline Zaretis isidora (Cramer 1779) & Zar_isi & & $\mathrm{x}$ & & & & & $\mathrm{CB}$ \\
\hline \multicolumn{9}{|l|}{ Subfamilia Cyrestinae } \\
\hline Marpesia chiron (Fabricius 1775) & Mar_chi & & & $\mathrm{x}$ & & & & $\mathrm{BM}, \mathrm{AA}, \mathrm{BB}$ \\
\hline Marpesia livius (Kirby 1871) & Mar_liv & & & & & $\mathrm{x}$ & & \\
\hline \multicolumn{9}{|l|}{ Subfamilia Danainae } \\
\hline Callithomia lenea (Cramer 1779) & Cal_len & $\mathrm{x}$ & & $\mathrm{x}$ & & & & \\
\hline Ceratinia neso (Hübner 1806) & Cer_nes & $\mathrm{x}$ & & $\mathrm{x}$ & & $\mathrm{x}$ & & B \\
\hline Ceratinia poecila (Bates 1862) & Cer_poe & $\mathrm{x}$ & & $\mathrm{x}$ & & $\mathrm{x}$ & & \\
\hline Dircenna adina (Hewitson 1855) & Dir_adi & $\mathrm{x}$ & & $\mathrm{x}$ & & & & BS, BB \\
\hline Dircenna loreta (Haensch 1903) & Dir lor & $\mathrm{x}$ & $\mathrm{x}$ & $\mathrm{x}$ & & & & \\
\hline Episcada clausina (Hewitson 1876) & Epi_cla & & & $\mathrm{x}$ & & & & \\
\hline Hypoleria asellia (Hopffer 1874) & Hyp_ase & $\mathrm{x}$ & & $\hat{x}$ & & & & \\
\hline Hypoleria sarepta (Hewitson 1852) & Hyp_sar & $\mathrm{x}$ & & $\mathrm{x}$ & & & & SB \\
\hline
\end{tabular}


Tabla 3. Continuación,

Table 3. Continuation.

\begin{tabular}{|c|c|c|c|c|c|c|c|c|}
\hline \multirow[b]{2}{*}{ Especie } & \multirow[b]{2}{*}{ Abreviatura } & \multicolumn{2}{|c|}{$\mathrm{BP}$} & \multicolumn{2}{|l|}{ BS } & \multicolumn{2}{|l|}{ C } & \multirow[b]{2}{*}{ Hábitat $^{1}$} \\
\hline & & $\mathrm{R}$ & $\mathrm{T}$ & $\mathrm{R}$ & $\mathrm{T}$ & $\mathrm{R}$ & $\mathrm{T}$ & \\
\hline \multicolumn{9}{|l|}{ Subfamilia Danainae } \\
\hline Hypothyris euclea (Godart 1819) & Hyp_euc & $\mathrm{x}$ & & $\mathrm{x}$ & & $\mathrm{x}$ & & \\
\hline Mechanitis messenoides (Felder and Felder 1865) & Mech_mes & & & $\mathrm{x}$ & & & & \\
\hline Mechanitis polymnia (Linnaeus 1758) & Mech_pol & $\mathrm{x}$ & & $x$ & & $\mathrm{x}$ & & $\mathrm{SB}, \mathrm{AA}$ \\
\hline Melinaea marsaeus (Hewitson 1860) & Mel_mar & & & & & & & \\
\hline Napeogenes inachia (Hewitson 1855) & Nap_ina & $\mathrm{x}$ & & $\mathrm{x}$ & & $\mathrm{x}$ & & $\mathrm{SB}, \mathrm{CB}$ \\
\hline Oleria victorine (Guérin-Méneville 1844) & Ole_vic & $\mathrm{x}$ & $\mathrm{x}$ & $\mathrm{x}$ & & $\mathrm{x}$ & & \\
\hline Tithorea harmonia cf. (Cramer 1777) & Tit_harm & $\mathrm{x}$ & & & & $x$ & & \\
\hline \multicolumn{9}{|l|}{ Subfamilia Heliconiinae } \\
\hline Eueides isabella (Stoll 1781) & Eue_isa & & & $\mathrm{x}$ & & $\mathrm{x}$ & & $\mathrm{BB}, \mathrm{P}$ \\
\hline Heliconius erato (Linnaeus 1758) & Hel_era & $x$ & & $\mathrm{x}$ & & $x$ & & \\
\hline Heliconius wallacei (Reakirt 1866) & Hel_wal & & & & $x$ & & & BS \\
\hline \multicolumn{9}{|l|}{ Subfamilia Limenitidinae } \\
\hline Adelpha capucinus (Walch 1775) & Ade_cap & & & & & $\mathrm{x}$ & $\mathrm{x}$ & $\mathrm{BM}, \mathrm{BS}, \mathrm{P}$ \\
\hline Adelpha cocala (Cramer 1779) & Ade_coc & $\mathrm{x}$ & & & & & & $\mathrm{BB}$ \\
\hline Adelpha iphiclus (Linnaeus 1758 ) & Ade_iph & & $x$ & & & & & BS, D \\
\hline Adelpha mesentina (Cramer 1777) & Ade_mes & & & $\mathrm{x}$ & & & & \\
\hline \multicolumn{9}{|l|}{ Subfamilia Nymphalinae } \\
\hline Colobura annulata (Willmott, Constantino y Hall 2001) & Col_ann & & $\mathrm{x}$ & $\mathrm{x}$ & $\mathrm{x}$ & & $\mathrm{x}$ & $\mathrm{B}$ \\
\hline Eresia clio (Linnaeus 1758) & Ere_cli & & & $\mathrm{x}$ & & $\mathrm{x}$ & & \\
\hline Eresia eunice (Hübner 1807) & Ere_eun & $\mathrm{x}$ & & & & & & \\
\hline Historis acheronta (Fabricius 1775) & His_ach & & & & & & $x$ & $\mathrm{P}^{*}$ \\
\hline Junonia genoveva (Felder and Felder 1867) & Jun_gen & & & & & $\mathrm{x}$ & $x$ & $\mathrm{CS}, \mathrm{P}^{*}$ \\
\hline Smyrna blomfildia (Fabricius 1781) & Smy_blo & & $x$ & & & & $\mathrm{x}$ & $\mathrm{BM}, \mathrm{BB}, \mathrm{CS}$ \\
\hline Tigridia acesta (Linnaeus 1758) & Tig_ace & & & $\mathrm{x}$ & $\mathrm{x}$ & & & $\mathrm{BM}, \mathrm{BB}, \mathrm{D}$ \\
\hline \multicolumn{9}{|l|}{ Subfamilia Satyrinae } \\
\hline Antirrhea philaretes (Felder and Felder 1862) & Ant_phi & $\mathrm{x}$ & $x$ & & & & & $\mathrm{~B}$ \\
\hline Bia rebeli (Bryk 1953) & Bia_reb & & & $\mathrm{x}$ & $\mathrm{x}$ & & & \\
\hline Caeruleuptychia glauca (Weymer 1911) & Cae_gla & & $\mathrm{x}$ & & $\mathrm{x}$ & & $\mathrm{x}$ & \\
\hline Caeruleuptychia urania (Butler 1867) & Cae_ura & $\mathrm{x}$ & & & & & $\mathrm{x}$ & \\
\hline Caligo illioneus (Cramer 1775) & Cal_ili & $\mathrm{x}$ & & $\mathrm{x}$ & & & & BM, BS, C \\
\hline Catoblepia berenchyntia (Stichel 196) & Cat_ber & $\mathrm{x}$ & $\mathrm{x}$ & & & & & SB \\
\hline Chloreuptychia herseis (Godart 1824) & Chl_her & & $\mathrm{x}$ & $\mathrm{x}$ & $\mathrm{x}$ & & & B \\
\hline Chloreuptychia tolumnia (Cramer 1777) & Chl_tol & & & $\mathrm{x}$ & & & & B \\
\hline Cissia myncea (Cramer 1780) & Cis_myn & & & $\mathrm{x}$ & & $\mathrm{x}$ & & B \\
\hline Cissia proba (Weymer 1911) & Cis_pro & & & & & $\mathrm{x}$ & $\mathrm{x}$ & B \\
\hline Cissia terrestris (Butler 1867) & Cis_ter & & $\mathrm{x}$ & $x$ & $\mathrm{x}$ & & & $\mathrm{B}$ \\
\hline Cithaerias aurorina (Weymer 1910) & Cith_aur & $\mathrm{x}$ & & & & & & $\mathrm{BM}^{*}$ \\
\hline Eryphanis lycomedon (Felder and Felder 1862) & Ery_lyc & & & $\mathrm{x}$ & & & & B \\
\hline Haetera piera (Linnaeus 1758) & Hae_pie & $\mathrm{x}$ & & $\mathrm{x}$ & & & & $\mathrm{BM}^{*}$ \\
\hline Harjesia obscura (Butler 1867) & Har_obs & & & & $x$ & & $x$ & $\mathrm{P}$ \\
\hline Hermeuptychia hermes (Fabricius 1775) & Her_her & & $\mathrm{x}$ & $\mathrm{x}$ & & $\mathrm{x}$ & $x$ & $\mathrm{AA}, \mathrm{P}^{*}$ \\
\hline Hermeuptychia sp. & Her_sp & & $x$ & $x$ & $x$ & $x$ & $x$ & \\
\hline Incertae sedis nerita (Capronier 1881) & Inc_sed. & & & $\mathrm{x}$ & $\mathrm{x}$ & $\mathrm{x}$ & $\mathrm{x}$ & \\
\hline Magneuptychia mimas (Godman 1905) & Mag_mim & & $x$ & & $\mathrm{x}$ & & $x$ & \\
\hline Magneuptychia moderata (Weymer 1911) & Mag_mod & & & $\mathrm{x}$ & & & & \\
\hline Magneuptychia nebulosa (Butler 1867) & Mag_neb & & & & $x$ & & $\mathrm{x}$ & \\
\hline Magneuptychia ocnus (Butler 1867) & Mag_ocn & $x$ & & & & & $\mathrm{x}$ & \\
\hline Megeuptychia antonoe (Cramer 1775) & Meg_ant & $x$ & $x$ & & & $\mathrm{x}$ & & $\mathrm{BB}, \mathrm{D}$ \\
\hline Morpho achilles (Linnaeus 1758) & Mor_ach & & & $x$ & & & & $\mathrm{SB}^{*}$ \\
\hline Morpho helenor (Cramer 1776) & Mor_hel & $\mathrm{x}$ & $\mathrm{x}$ & $\mathrm{x}$ & & & & $\mathrm{BM}, \mathrm{BS}, \mathrm{CB}$ \\
\hline Opsiphanes cassina (Felder and Felder 1862) & Ops_cas & & $x$ & & $\mathrm{x}$ & & $\mathrm{x}$ & $\mathrm{AA}$ \\
\hline Opsiphanes invirae (Hübner 1808) & Ops_inv & $\mathrm{x}$ & & & & $\mathrm{x}$ & & $\mathrm{D}^{*}$ \\
\hline Pareuptychia hesionides (Foster 1964) & Par_hes & $\mathrm{x}$ & $\mathrm{x}$ & $\mathrm{x}$ & $\mathrm{x}$ & $x$ & $\mathrm{x}$ & \\
\hline Pierella hyceta ceryce (Hewitson 1859) & Pie_hyc & $\mathrm{x}$ & & $\mathrm{x}$ & & & & $\mathrm{BM}$ \\
\hline Pierella lena (Constantino 2007) & Pie_len & $x$ & & & & & & $\mathrm{BM}, \mathrm{CB}$ \\
\hline Pseudodebis celia (Cramer 1779) & Pse_cel & & & & $\mathrm{x}$ & & & \\
\hline Pseudodebis valentina (Cramer 1779) & Pse_val & $\mathrm{x}$ & $\mathrm{x}$ & $\mathrm{x}$ & $x$ & $\mathrm{x}$ & $x$ & BM, SB \\
\hline Taygetina kerea (Butler 1869) & Tay_ker & & $x$ & & & & & $\mathrm{CB}$ \\
\hline Taygetis laches (Fabricius 1973) & Tay_lac & & & $\mathrm{x}$ & & $\mathrm{x}$ & $\mathrm{x}$ & \\
\hline Taygetis mermeria (Cramer 1776) & Tay_mer & & $\mathrm{x}$ & $\mathrm{x}$ & $\mathrm{x}$ & & $x$ & $\mathrm{BM}^{*}$ \\
\hline Taygetis rufomarginata (Staudinger 1888) & Tay_ruf & $x$ & $\mathrm{x}$ & & $\mathrm{x}$ & $\mathrm{x}$ & $x$ & \\
\hline Taygetis sp. & Tay_sp & $x$ & & & & & & \\
\hline Taygetis sylvia (Bates 1866) & Tay_syl & & $\mathrm{x}$ & $\mathrm{x}$ & $\mathrm{x}$ & & $x$ & $\mathrm{CB}$ \\
\hline Yphtimoides renata (Stoll 1780) & Yph_ren & & & & & $x$ & $x$ & $\mathrm{BB}, \mathrm{P}$ \\
\hline Yphtimoides sp. & Yph_sp & & & & $\mathrm{x}$ & $x$ & $x$ & \\
\hline
\end{tabular}

${ }^{1}$ Hábitat según revisión de diversos estudios (literatura citada y DeVries 1987): AA=áreas abiertas, B=bosque, $\mathrm{BB}=$ borde de bosque, $\mathrm{BF}=$ bosque fragmentado, $\mathrm{BM}=$ bosque maduro, $\mathrm{BS}=$ bosque secundario, $\mathrm{C}=$ cultivo, $\mathrm{CB}=$ claro de bosque, $\mathrm{CS}=$ cultivo con sombra, $\mathrm{D}=$ dosel, $\mathrm{MA}=$ matriz agropecuaria, $\mathrm{P}=$ perturbado, $\mathrm{SB}=$ sotobosque, *=indicadora del tipo de hábitat. 
I. s. nerita, M. nebulosa, H. hermes, Taygetis sylvia y Temenis laothoe) fueron abundantes durante la época seca, pero poco comunes durante la época de transición. La presencia-ausencia de especies de mariposas fue bien explicada por las características del hábitat (nivel de perturbación, porcentaje de la cobertura herbácea), la época de año y la estructura espacial (MEM1) (pseudo- $F_{5,53}=1.46, P=0.001$, $\left.R_{\text {adi }}^{2}=0.04\right)$. El 55\% de la variación fue explicada exclusivamente por las características del hábitat, el $24 \%$ por la época y solo el $14 \%$ por la estructura espacial. El CCA1 se asoció con el gradiente de perturbación (desde cultivo hasta bosque primario) y el porcentaje de cobertura herbácea estuvo asociado con el nivel de perturbación. El CCA2 separó la época de transición y la época seca, y se asoció al efecto de la estructura espacial (Figura 4).

\section{Discusión}

La riqueza de especies (107 especies) y el porcentaje de especies representadas por un solo individuo (23\%) en este trabajo están en el rango reportado en otros estudios con Nymphalidae en bosques húmedos tropicales (sin tomar en cuenta el posible efecto por diferencias en área muestreada), que reportan 85-128 especies y 14-28\% de especies representadas por un individuo (DeVries et al. 1997; 2011; DeVries and Walla 2001; Hamer et al. 2006; Barlow et al. 2007; Grøtan et al. 2014). Este patrón también se registró en bosques amazónicos basimontanos cercanos al sitio de estudio, con 98-123 especies y 25-26\% de especies representadas por solo un individuo (Apaza 2005; Aliaga 2012; Identidad Madidi and SERNAP 2019). Aunque la frecuencia de especies con un individuo generalmente se asocia a especies raras, también podría tratarse de sub-representación por las características de comportamiento de la especie o fluctuaciones anuales en la abundancia de cada especie, que suelen ser comunes en esta familia (DeVries and Walla 2001; Grøtan et al. 2012, 2014). De acuerdo con las estimaciones de la riqueza en cada tipo de ambiente mediante el índice de Chao 1, el esfuerzo de muestreo realizado en este trabajo permitió tener una muestra representativa de las comunidades en cada uno de ellos (76-81\% de especies registradas), aunque sugiere que la comunidad observada en los cultivos de cacao es algo más incompleta (62 especies registradas de 82 estimadas). Sin embargo, es importante resaltar que el esquema de muestreo de este trabajo sólo permitió capturar mariposas a nivel de sotobosque. Es muy probable que la riqueza de mariposas en bosque primario y bosque secundario esté subrepresentada, ya que 17$25 \%$ de las especies de mariposas de la familia Nymphalidae de bosque se pueden registrar casi exclusivamente a nivel de dosel (DeVries 1988; DeVries et al. 1997; Barlow et al. 2007; Ribeiro and Freitas 2012).

La riqueza de especies, la abundancia y la heterogeneidad de mariposas en el sitio de estudio fue mayor durante la época seca. Varios estudios registraron la fluctuación estacional de la abundancia y la riqueza de especies de las comunidades de mariposas de la familia Nymphalidae, mostrando una correlación con la variación de la precipitación (Grøtan et al. 2012) y de la temperatura (Grøtan et al. 2014). Algunas comunidades en hábitats a menor altitud (e.g., bosque amazónico de llanura en Ecuador) presentan picos de diversidad en época húmeda o antes de ésta (DeVries et al. 1997; DeVries and Walla 2001), mientras que comunidades de bosques de llanura de Costa Rica (DeVries et al. 2011; Grøtan et al. 2012, 2014) muestran resultados similares a nuestro estudio (mayor riqueza y abundancia en época seca). El estudio realizado por Apaza (2005) en un área cercana a nuestro sitio de estudio reportó también un pico de riqueza en época seca (agosto) y una disminución hacia la época húmeda.

\section{Efecto de la perturbación}

La variación en la riqueza y abundancia de especies entre los niveles de perturbación comparados dependió de la época, lo que podría atribuirse a la temperatura. Cada especie de mariposa tiene un rango óptimo de temperatura para su actividad, cuya amplitud varía según el comportamiento y preferencia de microhábitat (Kingslover 1985; Kuchlein and Ellis 1997). La temperatura máxima promedio en el mes de agosto (época seca) es de $33^{\circ} \mathrm{C}$, mientras que en el mes de noviembre (transición) alcanza los $37{ }^{\circ} \mathrm{C}$ (SENAMHI 2020). Ese cambio es más drástico en sitios más expuestos, como los cultivos, ya que la cobertura de dosel permite mantener temperaturas más frescas durante la época más caliente (Chen et al. 1999; Lin 2007; Beserra et al. 2012; de Souza et al. 2012). Por ejemplo, de Souza et al. (2012) registraron una diferencia de $6{ }^{\circ} \mathrm{C}$ entre cultivos de café con y sin sombra. Entonces, es posible que durante la época de transición el efecto de la 
temperatura sea mayor en los cultivos de cacao y solo algunas especies que tiene preferencia por áreas abiertas (e.g., J. genoveva, Hamadryas februa, Hamadryas feronia) puedan aprovechar los recursos en esas condiciones (Beserra et al. 2012).

\section{Composición del ensamblaje}

La variación en la composición del ensamblaje de mariposas estuvo influenciada principalmente por el nivel de perturbación en los sitios muestreados, con una composición de especies distinta entre los cultivos y ambos tipos de bosque. En general, el bosque húmedo tropical es heterogéneo en tiempo y espacio (Connell 1978; Gentry 1992), y la heterogeneidad espacial es causada principalmente por perturbaciones naturales que, además de mantener diversos estados sucesionales, contribuyen a la alta diversidad de especies (Brown and Hutchings 1997; Schnitzer and Carson 2001; Pardonnet 2010). Las adaptaciones a la heterogeneidad espacial permiten el desplazamiento de mariposas entre fragmentos de bosque conservado y los sitios perturbados (Beck and Schulze 2000; Marini-Filho and Martins 2010), lo que genera similitudes en la composición de los ensamblajes. Esto puede explicar la presencia de especies conocidas como indicadoras de bosque primario en bosque secundario (e.g., Haetera piera, Pierella hyceta, Nessaea obrinus, Consul fabius, Bia rebeli y Morpho achilles) y en cultivos (Taygetis mermeria), así como la presencia de algunas especies de sotobosque en los cultivos (Oleria victorine y $P$. valentina). Otros estudios también reportaron la presencia de especies de bosque primario en sitios con mayor perturbación, y lo atribuyeron principalmente a la presencia de sus plantas hospederas (DeVries et al. 1997; Wood and Gillman 1998; Ghazoul 2002). También se reportó que la distancia entre fragmentos de bosque no afecta la presencia de mariposas tanto comolohacela disponibilidad de recursos alimenticios (Haddad and Baum 1999; Krauss et al. 2004; Veddeler et al. 2005). Las mariposas de borde de bosque o dosel son más tolerantes que las especies de sotobosque al incremento de radiación solar y temperatura que causa la perturbación (Warren 1985; Lovejoy et al. 1986; Brito et al. 2014). Esto concuerda con nuestros registros de especies de dosel, borde y claro de bosque (e.g., Memphis acidalia, M. glauce, C. neso, Hypothyris euclea, N. inachia y H. hermes) en cultivos. Aunque no se pueda asegurar su permanencia a largo plazo, es evidente que existen recursos y condiciones ambientales que permiten la presencia, al menos ocasional, de especies de bosque primario en estos cultivos (Horner-Devine et al. 2003; Ritter et al. 2011; Beserra et al. 2012).

La variación en la composición de los ensamblajes también estuvo determinada por la época y en menor medida por la estructura espacial. Esto significa que si bien la cercanía entre los sitios puede favorecer la predictibilidad de la presencia de una especie, las características del hábitat y de la época son más determinantes. El decremento en la abundancia durante la época de transición fue general, pero los cambios estacionales afectaron de manera diferente a cada especie (Kingslover 1985; Kuchlein and Ellis 1997; Cormont et al. 2011; Lourenço et al. 2019). Algunas especies (e.g., C. poecilia, M. nebulosa y Megeuptychia antonoe) tuvieron una reducción poblacional más drástica, pasando de ser especies abundantes en época seca a contar con solo un individuo en época de transición, mientras que otras especies como $M$. polymnia y H. feronia mostraron el patrón opuesto.

Entre las variables ambientales consideradas para este estudio, la cobertura herbácea (correlacionada inversamente con la cobertura de dosel) se asoció con la diferencia en la composición de especies entre niveles de perturbación, diferenciando a los cultivos de los dos tipos de bosque. En los cultivos se capturaron más especies que suelen habitar en pastizales y sitios abiertos (expuestos a la radiación solar). Sin embargo, la variación de los ensamblajes de mariposas podría estar mejor explicada por otras variables ambientales no medidas, como la presencia de plantas hospederas (Yamamoto et al. 2007), la riqueza, composición y estructura vertical de la vegetación (Zellweger et al. 2016; Graça et al. 2017) o la disponibilidad de recursos. Los principales recursos alimenticios para la familia Nymphalidae dependen de la época de floración y fructificación (para las especies nectarívoras y frugívoras, respectivamente) y varían de manera estacional (Brito et al. 2014; Castro and Espinosa 2015). Aunque no cuantificamos la disponibilidad de recursos, fue muy evidente la presencia de recursos para ambos grupos en los tres niveles de perturbación durante la época seca (observación personal). En la época de transición, en cambio, las plantas cultivadas ya no tenían frutos, pero en los sitios con bosque aún existían plantas en fructificación, lo que 
explica la mayor disminución en abundancia y especies en los cultivos. Por otro lado, en los cultivos había plantas en floración en ambas épocas (observación personal), lo que favorecería la presencia de mariposas nectarívoras tolerantes a la radiación solar en la época de transición (Harvey et al. 2006). Esto fue particularmente evidente para J. genoveva, una de las especies dominantes en este ambiente y cuya dominancia explica la reducción en la heterogeneidad en el cultivo (el índice de heterogeneidad es sensible a la abundancia [Magurran 2005]).

\section{Efecto del método de muestreo}

Es importante notar que el método de muestreo podría tener un papel clave en la evaluación del impacto de la perturbación sobre las mariposas de la familia Nymphalidae. Las trampas de cebo son muy efectivas para mariposas frugívoras (Graça et al. 2017), mientras que las redes son efectivas tanto para frugívoras como para nectarívoras (Castro and Espinosa 2015). Harvey et al. (2006) encontraron mayor riqueza y abundancia de mariposas frugívoras en niveles de perturbación media en comparación a sitios muy abiertos, pero no hallaron diferencias entre niveles de perturbación para mariposas nectarívoras ni para el ensamblaje completo. En nuestro estudio sólo se detectó una variación significativa entre los niveles de perturbación con el método de captura manual: durante la época de transición, la abundancia y la heterogeneidad fueron mayores en bosque primario que en cultivo. Esto puede deberse a que dos de las especies más abundantes en cultivo (J. genoveva y Hermeuptychia sp.) fueron capturadas mayormente por el método manual (91\% y 70\%, respectivamente), reduciendo la heterogeneidad en este nivel de perturbación. Dado que la perturbación puede afectar de distinta manera a distintos grupos del ensamblaje de mariposas, es importante que las evaluaciones complementen el muestreo con captura manual (Wood and Gillman 1998; Castro and Espinosa 2015; Jakubikova and Kadlec 2015; Graça et al. 2017; Checa et al. 2019).

\section{Conclusiones}

La presencia de especies asociadas a bosque conservado en el área de estudio, sumada a la similitud en la composición de sitios de bosque primario y secundario, resalta la importancia del bosque primario para conservar la riqueza de Nymphalidae en el área (Wood and Gillman 1998; Horner-Devine et al. 2003; Veddeler et al. 2005; Gibson et al. 2011). Ekroos et al. (2010) resaltan la susceptibilidad de las especies especialistas a la modificación del hábitat y, aunque registramos especies especialistas de bosque en el nivel de perturbación medio (bosque secundario), los sitios evaluados fueron abandonados hace al menos 20 años (Seidel en prensa). Si la dinámica de perturbación se intensifica, es posible que estas especies se extingan localmente (Brown and Hutchings 1997; Schulze et al. 2004).

Los resultados de nuestro estudio muestran que para evaluar el impacto de la perturbación sobre poblaciones de mariposas, además del nivel de perturbación se debe realizar la partición de los datos según época del año. Aunque varios estudios enfocados en el efecto de la perturbación efectúan un muestreo temporal continuo de tres meses o más, realizan la evaluación de la perturbación con los datos conglomerados (e.g., Lewis 2001; Tobar et al. 2006; Tórrez et al. 2013; Gonzáles-Valdivia et al. 2016) o sólo realizan la toma de datos en una época (e.g., Harvey et al. 2006), lo cual puede generar una dilución de la detección del efecto de la perturbación sobre las comunidades. Además, es importante utilizar métodos de muestreo complementarios para evaluar el efecto sobre diferentes gremios alimentarios. Es necesario tomar en cuenta que la composición de mariposas puede estar siendo afectada por otras variables ambientales, principalmente aquellas relacionadas con la estructura de la vegetación, la disponibilidad de recursos y las diferencias en la temperatura que se alcanza en cada tipo de hábitat. Hasta donde tenemos conocimiento, este es el primer estudio que evalúa el efecto del cambio de uso del suelo sobre la fauna de lepidópteros en Bolivia, con un diseño de muestreo cuasi-experimental y utilizando un enfoque estadístico que permite evaluar integralmente las variables asociadas a la sensibilidad de este grupo de mariposas.

Agradecimientos. La investigación fue realizada en el marco del proyecto "Investigación de la Biodiversidad como base para el manejo de recursos naturales en Alto Beni", financiado con fondos IDH (Impuesto Directo a los Hidrocarburos) - UMSA. Los autores agradecen a C. Molina, A. Céspedes, R. López, D. Larrea, B. Huertas, F. Milesi y dos revisores anónimos por sus comentarios sobre el manuscrito, y a los trabajadores 
del proyecto "Comparación a largo plazo de sistemas agrícolas en los trópicos para la producción del cacao (Theobroma cacao L.)", a cargo del Instituto de Investigaciones para la Agricultura Orgánica (FiBL: Forschungs Institutfür Biologischen Landbau), Frick, Suiza, por el apoyo logístico brindado durante el trabajo en campo.

\section{REFERENCIAS}

Aliaga, J. C. 2012. Estudio de la diversidad de lepidópteros diurnos en agroecosistemas de la colonia Villa Camacho, Sud Yungas, La Paz. Licenciatura en Ingeniería Agronómica, Universidad Mayor de San Andrés, La Paz. Bolivia. Pp. 173.

Altermatt, F. 2012. Temperature-related shifts in butterfly phenology depend on the habitat. Global Change Biology 18:2429-2438. https://doi.org/10.1111/j.1365-2486.2012.02727.x.

Apaza, M. 2005. Evaluación del grado de amenaza al hábitat a través de bioindicadores (lepidópteros) en dos comunidades del área de influencia del Parque Nacional y Área Natural de Manejo Integrado Madidi, La Paz, Bolivia. Licenciatura en Ingeniería Agronómica, Universidad Mayor de San Andrés, La Paz. Bolivia. Pp. 181.

Araujo, F. R. 2000. Nymphalid butterfly communities in an Amazonian forest fragment. Journal of Research on the Lepidoptera 35:29-41.

Barlow, J., W. L. Overal, I. S. Araujo, T. A. Gardner, and C. A. Peres. 2007. The value of primary, secondary and plantation forests for fruit-feeding butterflies in the Brazilian Amazon. Journal of Applied Ecology 44:1001-1012. https://doi.org/10.1111/j.1365-2664.2007.01347.x.

Barton, K. 2019. MuMIn: Multi-Model Inference. R package version 1.43.15. URL: CRAN.R-project.org/ package=MuMIn.

Bates, D., M. Maechler, B. Bolker, and S. Walker. 2015. Fitting Linear Mixed-Effects Models Using lme4. Journal of Statistical Software 67:1-48. https://doi.org/10.18637/jss.v067.i01.

Beccaloni, G. W., and K. J. Gaston. 1995. Predicting the species richness of neotropical forest butterflies: Ithomiinae (Lepidoptera: Nymphalidae) as indicators. Biological Conservation 71:77-86. https://doi.org/10.1016/00063207(94)00023-J.

Beck, J., and C. H. Schulze. 2000. Diversity of fruit-feeding butterflies (Nymphalidae) along a gradient of tropical rainforest succession in Borneo with some remarks on the problem of "pseudoreplicates". Lepidoptera Science 51: 89-98. https://doi.org/10.18984/lepid.51.2_89.

Beserra, C. E., L. Iannuzzi, and C. Schlindwein. 2012. Seasonality of fruit-feeding butterflies (Lepidoptera, Nymphalidae) in a Brazilian semiarid area. International Scholarly Research Network Zoology 2012:1-8. https://doi.org/10.5402/ 2012/268159.

Borcard, D., F. Gillet, and P. Legendre. 2018. Numerical ecology with R. Second edition. Springer, New York, USA. https://doi.org/10.1007/978-3-319-71404-2.

Brito, M. M., D. B. Ribeiro, M. Raniero, E. Hasui, F. N. Ramos, and A. Arab. 2014. Functional composition and phenology of fruit-feeding butterflies in a fragmented landscape: variation of seasonality between habitat specialists. Journal of Insect Conservation 18:547-560. https://doi.org/10.1007/s10841-014-9650-8.

Brown, K. S., and R. W. Hutchings. 1997. Disturbance, fragmentation, and the dynamics of diversity in Amazonian forest butterflies. Pp. 91-110 en W. F. Laurance and R. O. Bierregaards (eds.). Tropical forest remnants: ecology, management, and conservation of fragmented communities. The University of Chicago Press, Chicago, USA.

Brown, S., and A. E. Lugo. 1990. Tropical secondary forests. Journal of Tropical Ecology 6:1-32. https://doi.org/10.1017/ S0266467400003989.

Burnham, K. P., and D. R. Anderson. 2002. Model selection and multimodel inference: a practical information-theoretic. Second edition. Springer, New York, USA.

Campos, C., S. Paredes, N. Chapi, and R. Seidel. 2010. Evaluación de la vegetación arbórea del bosque de Sara Ana. Pp. 108-125 en S. G. Beck, N. Paniagua, N. Nagashiro and R. P. López (eds.). Biodiversidad y ecología en Bolivia - Simposio de los 30 años del Instituto de ecología. Instituto de Ecología, La Paz, Bolivia.

Castro, A., and C. I. Espinosa. 2015. Seasonal diversity of butterflies and its relationship with woody-plant resources availability in an Ecuadorian tropical dry forest. Tropical Conservation Science 8:333-351. https://doi.org/10.1177/ 194008291500800205.

Checa, M. F., D. A. Donoso, J. Rodríguez, E. Levy, A. Warren, and K. Willmott. 2019. Combining sampling techniques aids monitoring of tropical butterflies. Insect Conservation and Diversity12:362-372. https://doi.org/10.1111/icad.12328.

Chen, J., S. C. Saunders, T. R. Crow, R. J. Naiman, K. D. Brosofske, G. D. Mroz, B. L. Brookshire, and J. F. Franklin. 1999. Microclimate in forest ecosystem and landscape ecology: variations in local climate can be used to monitor and compare the effects of different management regimes. BioScience 49:288-297. https://doi.org/10.2307/1313612.

Chiu, C.-H., Y.-T. Wang, B. A. Walther, and A. Chao. 2014. An improved nonparametric lower bound of species richness via a modified good-turing frequency formula. Biometrics 70:671-682. https://doi.org/10.1111/biom.12200.

Connell, J. H. 1978. Diversity in tropical rain forests and coral reefs. Science 199:1302-1310. https://doi.org/10.1126/ science.199.4335.1302.

Corlett, R. T. 1995. Tropical secondary forests. Progress in Physical Geography 19:159-172. https://doi.org/10.1177/ 030913339501900201.

Cormont, A., A. H. Malinowska, O. Kostenko, V. Radchuk, L. Hemerik M. F. WallisDeVries, and J. Verboom. 2011. Effect of local weather on butterfly flight behavior, movement, and colonization: significance for dispersal under climate change. Biodiversity and Conservation 20:483-503. https://doi.org/10.1007/s10531-010-9960-4. 
de Souza, H. N., R. G. de Goede, L. Brussaard, I. M. Cardoso, E. M. Duarte, R. B. Fernandes, L. C. Gomes, and M. M. Pulleman. 2012. Protective shade, tree diversity and soil properties in coffee agroforestry systems in the Atlantic Rainforest biome. Agriculture, Ecosystems and Environment 146:176-196.https://doi.org/10.1016/j.agee.2011.11.007.

DeVries, P. J. 1987. The butterflies of Costa Rica and their natural history: Papilionidae, Pieridae, and Nymphalidae. Princeton University Press. Princeton. USA.

DeVries, P. J. 1988. Stratification of fruit-feeding nymphalid in a Costa Rican rainforest. Journal of Research on the Lepidoptera 26:98-108.

DeVries, P. J., L. G. Alexander, I. A. Chacon, and J. A. Fordyce. 2011. Similarity and difference among rainforest fruit-feeding butterfly communities in Central and South America. Journal of Animal Ecology 81:472-482. https: //doi.org/10.1111/j.1365-2656.2011.01922.x

DeVries, P. J., D. Murray, and R. Lande. 1997. Species diversity in vertical, horizontal, and temporal dimensions of a fruit-feeding butterfly community in an Ecuadorian rainforest. Biological Journal of the Linnean Society 62:343-364. https://doi.org/10.1006/bijl.1997.0155.

DeVries, P. J., and T. R. Walla. 2001. Species diversity and community structure in neotropical fruit-feeding butterflies. Biological Journal of the Linnean Society 74:1-15. https://doi.org/10.1006/bij1.2001.0571.

Dray, S., P. Legendre, and P. R. Peres-Neto. 2006. Spatial modeling: a comprehensive framework for principal coordinate analysis of neighbor matrices (PCNM). Ecological Modeling 196:483-493. https://doi.org/10.1016/ j.ecolmodel.2006.02.015.

Dray, S., D. Bauman, G. Blanchet, D. Borcard, S. Clappe, G. Guenard, T. Jombart, G. Larocque, P. Legendre, N. Madi, and H. H. Wagner. 2019. adespatial: Multivariate Multiscale Spatial Analysis. R package version 0.3-7. URL: CRAN.Rproject.org/package=adespatial.

Ekroos, J., J. Heliölä, and M. Kuussaari. 2010. Homogenization of lepidopteran communities in intensively cultivated agricultural landscapes. Journal of Applied Ecology 47:459-467. https://doi.org/10.1111/j.1365-2664.2009.01767.x.

FAO. 2020a. Forestal extent and changes. Pp. 11-24 en FAO. Global Forest Resources Assessment 2020: Main report. Food and Agriculture Organization of the United Nations. Rome, Italy.

FAO. 2020b. Evaluación de los recursos forestales mundiales 2020. Informe: Estado Plurinacional de Bolivia. Organización de las Naciones Unidas para la Alimentación y la Agricultura. Roma, Italia.

Faraway, J. J. 2016. Extending the linear model with R: Generalized linear, mixed effects and nonparametric regression models. Second edition. CRC Press, Boca Ratón, Florida, USA. https://doi.org/10.1201/b21296.

Fox, J., and S. Weisberg. 2019. An R Companion to Applied Regression, Third edition. Thousand Oaks CA: Sage.

Galipaud, M., M. A. Gillingham, and F. X. Dechaume-Moncharmont. 2017. A farewell to the sum of Akaike weights: the benefits of alternative metrics for variable importance estimations in model selection. Methods in Ecology and Evolution 8:1-11. https://doi.org/10.1111/2041-210X.12835.

Gareca, Y., E. Forno, T. W. Pyrcz, K. R. Willmott, and S. Reichle. 2006. Mariposas diurnas de Bolivia. Prometa, Santa Cruz, Bolivia.

Gascon, C., T. E. Lovejoy, R. O. Bierregaard, J. R. Malcolm, P. C. Stouffer, H. L. Vasconcelos, W. F. Laurance, B. Zimmerman, M. Tocher, and S. Borges. 1999. Matrix habitat and species richness in tropical forest remnants. Biological Conservation 91:223-229. https://doi.org/10.1016/S0006-3207(99)00080-4.

Gentry, A. H. 1992. Tropical forest biodiversity: distributional patterns and their conservational significance. Oikos 63:19-28. https://doi.org/10.2307/3545512.

Ghazoul, J. 2002. Impact of logging on the richness and diversity of forest butterflies in a tropical dry forest in Thailand. Biodiversity and Conservation 11:521-541. https://doi.org/10.1023/A:1014812701423.

Gibson, L., T. M. Lee, L. P. Koh, B. W. Brook, T. A. Gardner, J. Barlow, C. A. Peres, C. J. Bradshaw, W. F. Laurance, T. E. Lovejoy, and N. S. Sodhi. 2011. Primary forests are irreplaceable for sustaining tropical biodiversity. Nature 478: 378-383. https://doi.org/10.1038/nature10425.

Giam, X., and J. D. Olden. 2016. Quantifying variable importance in a multimodel inference framework. Methods in Ecology and Evolution 7:388-397. https://doi.org/10.1111/2041-210X.12492.

González-Valdivia, N. A., C. Pozo, S. Ochoa-Gaona, B. G. Ferguson, E. Cambranis, O. Lara, I. Pérez-Hernández, A. Ponce-Mendoza, and C. Kampichler. 2016. Nymphalidae frugívoras (Lepidoptera: Papilionoidea) asociadas a un ecomosaico agropecuario y de bosque tropical lluvioso en un paisaje del sureste de México. Revista Mexicana de Biodiversidad 87:451-464. https://doi.org/10.1016/j.rmb.2016.04.003.

Graça, M. B., J. L. Souza, E. Franklin, J. W. Morais, and P. A. Pequeno. 2017. Sampling effort and common species: optimizing surveys of understory fruit-feeding butterflies in the Central Amazon. Ecological Indicators 73:181-188. https://doi.org/10.1016/j.ecolind.2016.09.040.

Grøtan, V., R. Lande, I. A. Chacon, and P. J. DeVries. 2014. Seasonal cycles of diversity and similarity in a Central American rainforest butterfly community. Ecography 37:509-516. https://doi.org/10.1111/ecog.00635.

Grøtan, V., R. Lande, S. Engen, B. E. Sæther, and P. J. DeVries. 2012. Seasonal cycles of species diversity and similarity in a tropical butterfly community: Seasonal cycles of species diversity. Journal of Animal Ecology 81:714-723. https: //doi.org/10.1111/j.1365-2656.2011.01950.x.

Guariguata, M. R., and R. Ostertag. 2001. Neotropical secondary forest succession: changes in structural and functional characteristics. Forest Ecology and Management 148:185-206. https://doi.org/10.1016/S0378-1127(00)00535-1.

Haddad, N. M., and K. A. Baum. 1999. An experimental test of corridor effects on butterfly densities. Ecological Applications 9:623-633. https://doi.org/10.1890/1051-0761(1999)009[0623:AETOCE]2.0.CO;2.

Hamer, K. C., J. K. Hill, S. Benedick, N. Mustaffa, T. N. Sherratt, and M. T. Maryati. 2003. Ecology of butterflies in natural and selectively logged forests of northern Borneo: the importance of habitat heterogeneity. Journal of Applied 
Ecology 40:150-162. https://doi.org/10.1046/j.1365-2664.2003.00783.x.

Hamer, K. C., J. K. Hill, S. Benedick, N. Mustaffa, V. K. Chey, and M. Maryati. 2006. Diversity and ecology of carrionand fruit-feeding butterflies in Bornean rain forest. Journal of Tropical Ecology 22:25-33. .

Hansen, M. C., P. V. Potapov, R. Moore, M. Hancher, S. A. Turubanova, A. Tyukavina, D. Thau, S. Stehman, S. Goetz, and T. R. Loveland. 2013. High-resolution global maps of 21st-century forest cover change. Science 342:850-853. https: //doi.org/10.1126/science.1244693.

Harvey, C. A., A. Medina, D. M. Sánchez, S. Vílchez, B. Hernández, J. C. Saenz, J. M. Maes, F. Casanoves, and F. L. Sinclair. 2006. Patterns of animal diversity in different forms of tree cover in agricultural landscapes. Ecological Applications 16:1986-1999. https://doi.org/10.1890/1051-0761(2006)016[1986:poadid]2.0.co;2.

Hill, M. O., and H. G. Gauch. 1980. Detrended correspondence analysis: an improved ordination technique. Vegetation 42:47-58. https://doi.org/10.1007/978-94-009-9197-2_7.

Hill, J. K., and K. C. Hamer. 2004. Determining impacts of habitat modification on diversity of tropical forest fauna: the importance of spatial scale. Journal of Applied Ecology 41:744-754. https://doi.org/10.1111/j.0021-8901.2004.00926.x.

Horner-Devine, M. C., G. C. Daily, P. R. Ehrlich, and C. L. Boggs. 2003. Countryside biogeography of tropical butterflies. Conservation Biology 17:168-177. https://doi.org/10.1046/j.1523-1739.2003.01310.x.

Hothorn, T., F. Bretz, and P. Westfall. 2008. Simultaneous inference in general parametric models. Biometrical Journal 50:346-363. https://doi.org/10.1002/bimj.200810425

Hoy, C. W., P. S. Grewal, J. L. Lawrence, G. Jagdale, and N. Acosta. 2008. Canonical correspondence analysis demonstrates unique soil conditions for entomopathogenic nematode species compared with other free-living nematode species. Biological Control 46:371-379. https://doi.org/10.1016/j.biocontrol.2008.06.001.

Identidad Madidi, and SERNAP. 2019. Informe de la expedición científica Identidad Madidi 2016. Relevamientos de biodiversidad en el Parque Nacional y Área Natural de Manejo Integrado Madidi. La Paz, Bolivia.

Jakubikova, L, and T. Kadlec. 2015. Butterfly bait traps versus zigzag walks: What is the better way to monitor common and threatened butterflies in non-tropical regions? Journal of Insect Conservation 19:911-919. https://doi.org/10.1007/ s10841-015-9809-y.

Jongman, R. H. G., C. J. ter Braak, and O. F. van Tongeren (eds.). 1995. Data analysis in community and landscape ecology. Cambridge University Press, Cambridge, UK. https://doi.org/10.1017/CBO9780511525575.

Kessler, M., S. Abrahamczyk, M. Bos, D. Buchori, D. D. Putra, S. R. Gradstein. P. Höhn, J. Kluge, F. Orend, R. Pitopang, S. Saleh, C. H. Schulze, S. G. Sporn, I. Steffan-Dewenter, S. S. Tjitrosoedirdjo, and T. Tscharntke. 2009. Alpha and beta diversity of plants and animals along a tropical land use gradient. Ecological Applications 19:2142-2156. https: //doi.org/10.1890/08-1074.1.

Kingslover, J. G. 1985. Butter'y thermoregulation: organismic mechanisms and population consequences. Journal of Research on the Lepidoptera 24:1-20.

Koh, L. P. 2007. Impacts of land-use change on South-east Asian forest butterflies: a review. Journal of Applied Ecology 44:703-713. https://doi.org/10.1111/j.1365-2664.2007.01324.x.

Krauss, J., I. Steffan-Dewenter, and T. Tscharntke. 2004. Landscape occupancy and local population size depends on host plant distribution in the butterfly Cupido minimus. Biological Conservation 120:355-361. https://doi.org/10.1016/ j.biocon.2004.03.007.

Kremen, C. 1992. Assessing the indicator properties of species assemblages for natural areas monitoring. Ecological Applications 2:203-217. https://doi.org/10.2307/1941776

Kremen, C., R. K. Colwell, T. L. Erwin, D. D. Murphy, R. A. Noss, and M. A. Sanjayan. 1993. Terrestrial arthropod assemblages: their use in conservation planning. Conservation Biology 7:796-808. https://doi.org/10.1046/j.15231739.1993.740796.x.

Kuchlein, J. H., and W. N. Ellis. 1997. Climate-induced changes in the microlepidoptera fauna of the Netherlands and the implications for nature conservation. Journal of Insect Conservation 1:73-80. https://doi.org/10.1023/A: 1018483026265.

Lawton, J. H., D. E. Bignell, B. Bolton, G. E Bloemers, P. Eggleton, P. M. Hammond, M. Hodda, R. D. Holt, T. B. Larsen, N. A. Mawdsley, N. E. Stork, D. S. Srivastava, and A. D. Watt. 1998. Biodiversity inventories, indicator taxa and effects of habitat modification in tropical forest. Nature 391:72-76. https://doi.org/10.1038/34166.

Lewis, O. 2001. Effect of experimental selective logging on tropical butterflies. Conservation Biology 15:389-400. https: //doi.org/10.1046/j.1523-1739.2001.015002389.x.

Lin, B. B. 2007. Agroforestry management as an adaptive strategy against potential microclimate extremes in coffee agriculture. Agricultural and Forest Meteorology 144:85-94. https://doi.org/10.1016/j.agrformet.2006.12.009.

Lourenço, G. M., P. Luna, R. Guevara, W. Dáttilo, A. V. L. Freitas, and S. P. Ribeiro. 2019. Temporal shifts in butterfly diversity: responses to natural and anthropic forest transitions. Journal of Insect Conservation 2019:1-11. https: //doi.org/10.1007/s10841-019-00207-0.

Lovejoy, T. E., R. O. Bierregaard, A. B. Rylands, J. R. Malcom, C. E. Quintela, L. H. Harper, K. S. Brown, A. H. Powell, G. V. Powell, H. O. Schubart, and M. B. Hays. 1986. Edge and other effects of isolation on Amazon forest fragments. Pp. 257-285 en M. E. Soules (ed.). Conservation biology: A science of scarcity and diversity. Sinauer Associates, USA.

Mackey, R. L., and D. J. Currie. 2001. The diversity-disturbance relationship: is it generally strong and peaked? Ecology 82:3479-3492. https://doi.org/10.1890/0012-9658(2001)082[3479:TDDRII]2.0.CO;2.

Magurran, A. E. 2005. Measuring Biological Diversity. Blackwell Science Ltd., Oxford, UK.

Marini-Filho, O. J., and R. P. Martins. 2010. Nymphalid butterfly dispersal among forest fragments at Serra da Canastra National Park, Brazil. Journal of Insect Conservation 14:401-411. https://doi.org/10.1007/s10841-010-9271-9.

Matteucci, S. D., and A. Colma. 1982. Metodología para el estudio de la vegetación. Vol. 22. Secretaría General de la 
Organización de los Estados Americanos, Washington DC, USA.

Mendenhall, C. D., D. S. Karp, C. F. Meyer, E. A. Hadly, and G. C. Daily. 2014. Predicting biodiversity change and averting collapse in agricultural landscapes. Nature 509:213-217. https://doi.org/10.1038/nature13139.

Mueller, R., S. G. Beck, and R. Lara. 2002. Vegetación potencial de los bosques de Yungas en Bolivia, basado en datos climáticos. Ecología en Bolivia 37:5-14.

Oksanen, J., F. G. Blanchet, M. Friendly, R. Kindt, P. Legendre, D. McGlinn, P. R. Minchin, R. B. O'Hara, G. L. Simpson, P. Solymos, M. H. Stevens, E. Szoecs, and H. Wagner. 2019. vegan: Community Ecology Package. R package version 2.5-6. URL: CRAN.R-project.org/package=vegan.

Pardonnet, S. 2010. Effect of tree-fall gaps on fruit-feeding Nymphalidae assemblages in a Peruvian rain forest. Master thesis. Department of physics, chemistry and biology, Linköpings University, Linköpings. Suecia. Pp. 17.

Perfecto, I., R. A. Rice, R. Greenberg, and M. E. Van der Voort. 1996. Shade coffee: a disappearing refuge for biodiversity: shade coffee plantations can contain as much biodiversity as forest habitats. BioScience 46:598-608. https://doi.org/ $10.2307 / 1312989$

R Core Team. 2019. R: A language and environment for statistical computing. R Foundation for Statistical Computing, Vienna, Austria. URL: R-project.org.

Ribeiro, D. B., and A. V. Freitas. 2012. The effect of reduced-impact logging on fruit-feeding butterflies in Central Amazon, Brazil. Journal of Insect Conservation 16:733-744. DOI 10.1007/s10841-012-9458-3.

Ribeiro, D. B., R. Batista, P. I. Prado, K. S. Brown, and A. V. Freitas. 2012. The importance of small scales to the fruitfeeding butterfly assemblages in a fragmented landscape. Biodiversity and Conservation 21:811-827. https://doi.org/ 10.1007/s10531-011-0222-x.

Richards, S. A. 2008. Dealing with overdispersed count data in applied ecology. Journal of Applied Ecology 45:218-227. https://doi.org/10.1111/j.1365-2664.2007.01377.x.

Ritter, C. D., R. Lemes, A. B. de Morais, and C. de Sales Dambros. 2011. Borboletas (Lepidoptera: Hesperioidea e Papilionoidea) de fragmentos de Floresta Ombrófila Mista, Rio Grande do Sul, Brasil. Biota Neotropica 11:1-8. https: //doi.org/10.1590/S1676-06032011000100033.

Schnitzer, S. A., and W. P. Carson. 2001. Treefall gaps and the maintenance of species diversity in a tropical forest. Ecology 82:913-919. https://doi.org/10.1890/0012-9658(2001)082[0913:TGATMO]2.0.CO;2.

Schulze, C. H., M. Waltert, P. J. Kessler, R. Pitopang, D. Veddeler, M. Mühlenberg, S. R. Gradstein, C. Leuschner, I. Steffan-Dewenter, and T. Tscharntke. 2004. Biodiversity indicator groups of tropical land-use systems: comparing plants, birds, and insects. Ecological Applications 14:1321-1333. https://doi.org/10.1890/02-5409.

Seidel, R., L. Marconi, O. Plata, C. Campos and S. Beck. En prensa. Vegetación de Sara Ana y sus alrededores. En M. Ripa and K. Naoki (eds.). Historia Natural de Sara Ana. Instituto de Ecología (IE). La Paz, Bolivia.

SENAMHI. 2020. SisMet: base de datos oficial del SENAMHI. Servicio Nacional de Meteorología e Hidrología, Bolivia. URL: senamhi.gob.bo/sismet.

Simpson, E. H. 1949. Measurement of diversity. Nature 163:688. https://doi.org/10.1038/163688a0.

Supp, S. R., and S. K. Ernest. 2014. Species-level and community-level responses to disturbance: a cross-community analysis. Ecology 95:1717-1723. https://doi.org/10.1890/13-2250.1.

Tobar, D. L., M. Ibrahim, and F. Casasola. 2006. Diversidad de mariposas en un paisaje agropecuario del Pacífico Central de Costa Rica. Agroforestería en las Américas 45:58-65.

Tórrez, M., W. Arendt, and J. M. Maes. 2013. Comunidades de aves y lepidópteros diurnos y las relaciones entre ellas en bosque nuboso y cafetal de Finca Santa Maura, Jinotega. Encuentro 95:69-79. https://doi.org/10.5377/ encuentro.v0i95.1225.

Vargas, E. A., L. F. Aguirre, M. I. Galarza, and E. Gareca. 2008. Ensamble de murciélagos en sitios con diferente grado de perturbación en un bosque montano del Parque Nacional Carrasco, Bolivia. Mastozoología Neotropical 15:297-308.

Veddeler, D., C. H. Schulze, I. Steffan-Dewenter, D. Buchori, and T. Tscharntke. 2005. The contribution of tropical secondary forest fragments to the conservation of fruit-feeding butterflies: effects of isolation and age. Biodiversity and Conservation 14:3577-3592. https://doi.org/10.1007/s10531-004-0829-2.

Venables, W. N., and B. D. Ripley. 2002. Modern Applied Statistics with S-PLUS. Fourth edition. Springer, New York, USA. https://doi.org/10.1007/978-0-387-21706-2.

Warren, A. D., K. J. Davis, E. M. Stangeland, J. P. Pelham, and N. V. Grishin. 2018. Illustrated lists of American butterflies. URL: www.butterfliesofamerica.com.

Warren, M. S. 1985. The influence of shade on butterfly numbers in woodland rides, with special reference to the wood white Leptidea sinapis. Biological Conservation 33:147-164. https://doi.org/10.1016/0006-3207(85)90101-6.

Wood, B., and M. P. Gillman. 1998. The effects of disturbance on forest butterflies using two methods of sampling in Trinidad. Biodiversity and Conservation 7:597-616. https://doi.org/10.1023/A:1008800317279.

Wright, S. J. 2005. Tropical forests in a changing environment. Trends in Ecology and Evolution 20:553-560. https: //doi.org/10.1016/j.tree.2005.07.009.

Wright, S. J., and H. C. Muller-Landau. 2006. The future of tropical forest species. Biotropica 38:287-301. https://doi.org/ 10.1111/j.1744-7429.2006.00154.x.

Yamamoto, N., J. Yokoyama, and M. Kawata. 2007. Relative resource abundance explains butterfly diversity in island communities. Proceedings of the National Academy of Sciences of the USA 104:10524-10529. https://doi.org/10.1073/ pnas.0701583104.

Zellweger, F., A. Baltensweiler, C. Ginzler, T. Roth, V. Braunisch, H. Bugmann, and K. Bollmann. 2016. Environmental predictors of species richness in forest landscapes: abiotic factors versus vegetation structure. Journal of Biogeography 43:1080-1090. https://doi.org/10.1111/jbi.12696. 